\title{
Parkinson's disease-linked Parkin mutations impair glutamatergic signaling in hippocampal neurons
}

\author{
Mei Zhu', Giuseppe P. Cortese $e^{1,2}$ and Clarissa L. Waites ${ }^{1,3,4^{*}}$ (I)
}

\begin{abstract}
Background: Parkinson's disease (PD)-associated E3 ubiquitin ligase Parkin is enriched at glutamatergic synapses, where it ubiquitinates multiple substrates, suggesting that its mutation/loss-of-function could contribute to the etiology of PD by disrupting excitatory neurotransmission. Here, we evaluate the impact of four common PD-associated Parkin point mutations (T240M, R275W, R334C, G430D) on glutamatergic synaptic function in hippocampal neurons.

Results: We find that expression of these point mutants in cultured hippocampal neurons from Parkin-deficient and Parkin-null backgrounds alters NMDA and AMPA receptor-mediated currents and cell-surface levels and prevents the induction of long-term depression. Mechanistically, we demonstrate that Parkin regulates NMDA receptor trafficking through its ubiquitination of GluN1, and that all four mutants are impaired in this ubiquitinating activity. Furthermore, Parkin regulates synaptic AMPA receptor trafficking via its binding and retention of the postsynaptic scaffold Homer1, and all mutants are similarly impaired in this capacity.
\end{abstract}

Conclusion: Our findings demonstrate that pathogenic Parkin mutations disrupt glutamatergic synaptic transmission in hippocampal neurons by impeding NMDA and AMPA receptor trafficking. Such effects may contribute to the pathophysiology of PD in PARK2 patients.

Keywords: Parkin, AMPAR, NMDAR, GluN1, Homer1, Parkinson's disease, Synapse

\section{Background}

Mutations in the PARK2 gene are the most common cause of autosomal recessive juvenile parkinsonism and a major contributor to familial and sporadic early-onset Parkinson's disease (PD) [1-4]. PARK2 encodes Parkin, a RING-between-RING domain E3 ubiquitin ligase that catalyzes the covalent attachment of ubiquitin to specific substrates and regulates vital cellular processes including mitochondrial quality control and apoptosis [5-7]. Although it remains unclear how Parkin loss-of-function precipitates the death of midbrain dopaminergic neurons to cause PD, its ubiquitination of mitochondrial proteins downstream of the kinase PINK1 has been shown to mediate mitophagy, a selective form of autophagy [8-11].

\footnotetext{
* Correspondence: cw2622@cumc.columbia.edu

${ }^{1}$ Department of Pathology and Cell Biology, Columbia University Medical Center, New York, NY 10032, USA

${ }^{3}$ Department of Neuroscience, Columbia University, New York, NY, USA

Full list of author information is available at the end of the article
}

The buildup of damaged and dysfunctional mitochondria promotes oxidative stress, to which dopaminergic neurons are particularly vulnerable [12, 13], potentially explaining one mechanism through which PARK2 mutations induce dopaminergic cell loss and the motor symptoms of PD.

However, Parkin is highly expressed throughout the brain and known to regulate other aspects of neuronal function, including glutamatergic neurotransmission [14]. Indeed, defects in glutamatergic transmission and plasticity are reported at hippocampal and corticostriatal synapses deficient in Parkin [15-20]. Parkin's mechanisms of action at excitatory synapses remain poorly understood, although its ubiquitinating activity has been found to regulate the stability and function of multiple synaptic substrates, including the presynaptic vesicle-associated protein synaptotagmins $\mathrm{XI}$ and IV, the postsynaptic scaffold PICK1, and the kainate receptor subunit GluK2 [21-26]. Furthermore, our recent work demonstrates that 
Parkin also has a structural role at the synapse, linking postsynaptic endocytic zones required for AMPA-type glutamate receptor (AMPAR) capture and internalization to the postsynaptic density through a direct interaction with the scaffold protein Homer1 [19]. In Parkin-deficient neurons, both the levels of postsynaptic Homer1 and the density of endocytic zones are significantly reduced, leading to impaired AMPAR retention at synapses and ultimately to decreased AMPAR-mediated currents [19].

Loss of these enzymatic and structural roles of Parkin at glutamatergic synapses likely contributes to the symptoms and progression of PD in patients with PARK2 mutations. Consistent with this concept, PD is recognized as a multi-system disorder with both motor and non-motor symptoms, including resting tremor, muscle rigidity, disordered sleep, sensory dysfunction, depression, and cognitive impairment $[27,28]$. Although some of the $>200$ pathogenic mutations, deletions, and exonic rearrangements identified in PARK2 have been shown to disrupt Parkin's E3 ligase activity [29-32], their effects at glutamatergic synapses, comprising the vast majority of synapses in the brain, are almost completely unexplored.

Here, we evaluate the effects of four PD-associated Parkin point mutations (T240M, R275W, R334C, G430D) on neurotransmission and plasticity in hippocampal neurons, which are both rich sources of glutamatergic synapses as well as critical substrates for learning and memory. We find that all four mutants alter NMDA- and AMPA-type glutamate receptor trafficking and signaling. Mechanistically, we identify NMDA receptor (NMDAR) subunit GluN1 as a novel Parkin substrate and find that the mutants are defective in GluN1 ubiquitination, leading to decreased cell-surface NMDAR levels. Furthermore, the mutants exhibit reduced binding and synaptic retention of Homer1, leading to decreased cell-surface AMPAR levels and impaired AMPAR internalization during the induction of synaptic depression. Taken together, these data demonstrate that pathogenic Parkin mutations impair glutamatergic neurotransmission and plasticity, likely contributing to the etiology of PARK2-associated PD.

\section{Results}

Pathogenic Parkin mutations alter AMPAR and NMDAR cell-surface levels and currents

More than 200 pathogenic mutations have been identified in PARK2, comprising point mutations, deletions, and exonic rearrangements that are associated with early-onset PD and autosomal recessive juvenile PD [5, 33, 34]. However, few of these have been evaluated for their impact on excitatory synapse function. We examined four of the most prevalent Parkin point mutations, collectively identified in $\sim 100$ families worldwide (Parkinson Disease Mutation Database) and located across three domains of Parkin (T240M and R275W in the RING1 domain; R334C in the IBR domain; G430D in the RING2 domain) [3, 35-37](Additional file 1: Figure S1a). We first assessed the expression and subcellular localization of these mutants in cultured hippocampal neurons from either the Parkin knockdown background (i.e., lentivirally transduced on 2 days in vitro (DIV) with an shRNA against rat Parkin (shParkin), previously shown to reduce Parkin levels by 50\% [19]), or Parkin knockout background (i.e., cultured from $\mathrm{KO}$ rats; SAGE/Horizon Discovery). GFP-tagged T240M, R275W, R334C, and G430D human Parkin constructs exhibited similar subcellular localizations to GFP-wild-type (WT) Parkin, and three of these mutants were expressed at similar levels (Fig. 1; Additional file 1: Figure S1b-d). G430D expression was somewhat higher in the knockdown background (Additional file 1: Figure S1b, d), perhaps reflecting a deficiency in Parkin auto-ubiquitination and degradation for this mutant.

We previously reported that Parkin knockdown/knockout decreased cell-surface AMPA receptor (AMPAR) levels, and that co-expression of WT-Parkin (shParkin-WT) rescued this phenotype to the level of GFP-expressing control neurons [19]. We therefore assessed whether expression of each of the four Parkin mutants could similarly rescue cell-surface AMPAR levels. As in our previous study, we observed that shParkin decreased GluA1 levels by nearly $20 \%$ compared to GFP or shParkin-WT expression (Fig. 1a, b). None of the four mutants could match WT Parkin restoration of GluA1 levels, although both T240M and R334C were able to partially rescue this phenotype (Fig. 1a, b). To rule out the ability of the mutants to act as dominant-negatives on endogenous Parkin, we also examined their effects in the KO background. Here, the mutants were similarly unable to increase surface GluA1 levels above those observed in the $\mathrm{KO}$ condition, while WT Parkin expression increased GluA1 levels by $20 \%$ (Fig. 1c, d), equivalent to its level of rescue in knockdown neurons (Fig. 1a, b). Moreover, expression of shParkin in the $\mathrm{KO}$ neurons did not further reduce or alter GluA1 levels (Fig. 1c, d), indicating its lack of off-target effects.

Given Parkin's reported ability to regulate other glutamate receptor types (e.g., kainate) via ubiquitination [26], we also examined cell-surface NMDA receptor levels in these neurons by immunostaining with a human-derived antibody against the GluN1 subunit [38]. Intriguingly, we observed that shParkin similarly reduced cell-surface GluN1 levels (by 15\%) compared to GFP or shParkin-WT expression (Fig. 2a, b). Again, three of the mutants (R334C, R275W, G430D) were unable to rescue this phenotype, while T240M rescued to the level of WT Parkin (Fig. 2a, b). These experiments were also performed in Parkin $\mathrm{KO}$ neurons, where it was confirmed that none of the 
a

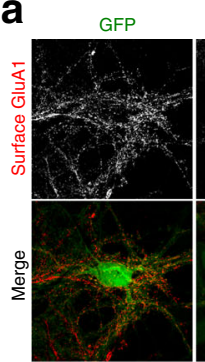

shParkin
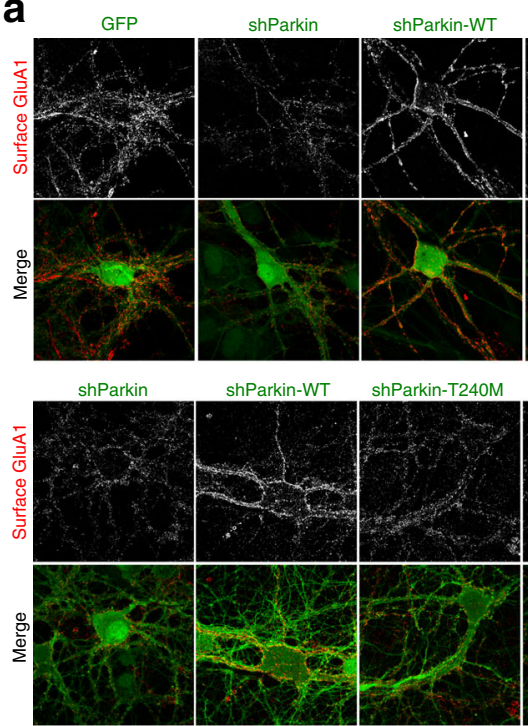

shParkin-T240M shParkin-R334C
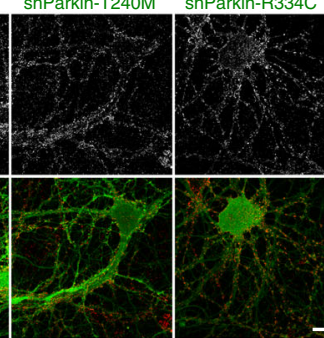

C

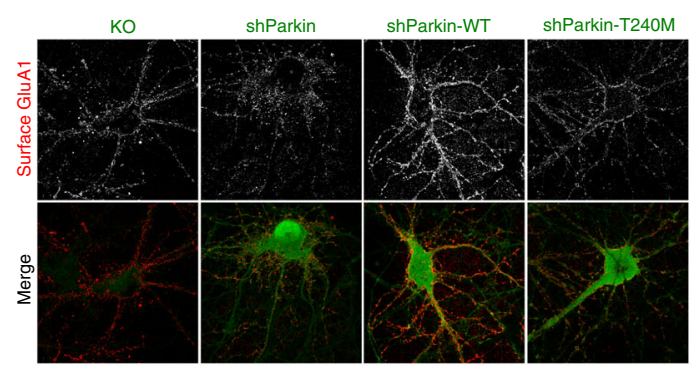

shParkin-R334C shParkin-R275W shParkin-G430D

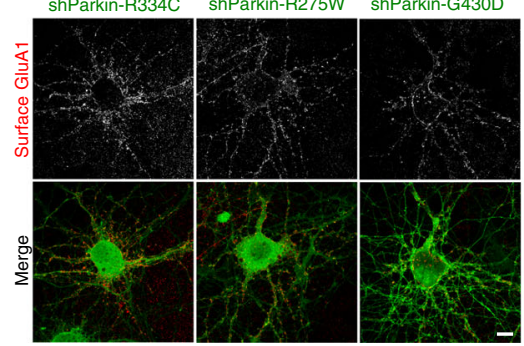

\section{b}

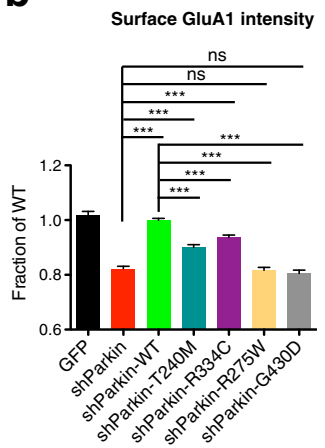

d

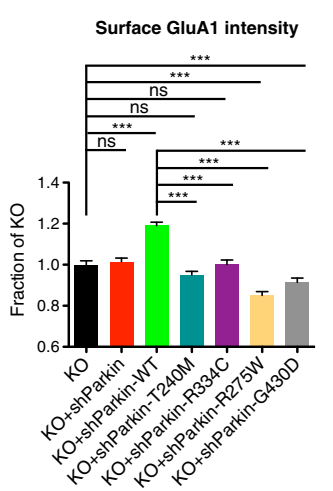

Fig. 1 Parkin mutation/loss-of-function decreases cell-surface GluA1 levels. a Representative images of surface GluA1 staining (red) in 14-16 DIV hippocampal neurons expressing GFP, shParkin, shParkin-WT, shParkin-T240M, shParkin-R275W, shParkin-R334C, or shParkin-G430D constructs. Scale bar, $10 \mu \mathrm{m}$. b Quantification of cell-surface GluA1 intensity expressed as a fraction of shParkin-WT ( $n \geq 70$ fields of view per condition with $>100$ GluA1 puncta per field, results confirmed in 4 independent experiments. ${ }^{* *} P<0.001$, one-way ANOVA, error bars represent SEM). c Representative images of surface GluA1 staining (red) in 14-16 DIV Parkin KO hippocampal neurons expressing shParkin, shParkin-WT, shParkin-T240M, shParkinR275W, shParkin-R334C, or shParkin-G430D constructs and non-transduced KO control. Scale bar, $10 \mu \mathrm{m}$. d Quantification of cell-surface GluA1 intensity expressed as a fraction of Parkin $\mathrm{KO}(n \geq 50$ fields of view per condition with $>100 \mathrm{GluA} 1$ puncta per field, results confirmed in 2 independent experiments. ${ }^{* *} P<0.001$, one-way ANOVA, error bars represent SEM)

mutants, including T240M, was able to increase surface GluN1 levels above those observed in the $\mathrm{KO}$ or $\mathrm{KO}+$ shParkin conditions (Fig. 2c, d). In contrast, WT Parkin expression increased GluN1 levels by 20\%, similar to its level of rescue in shParkin-expressing neurons (Fig. 2c, d). Together, these findings indicate that none of the four Parkin mutants are able to support normal cell-surface AMPAR and NMDAR levels.
To determine whether the Parkin mutants compromise excitatory neurotransmission, we examined their effects on AMPA and NMDA receptor spontaneous excitatory postsynaptic currents (EPSCs) in both Parkin knockdown and KO neurons. We compared the degree of rescue from expression of Parkin mutants to that of WT Parkin, which significantly increased AMPAR miniature EPSC (mEPSC) amplitudes in the knockdown and 

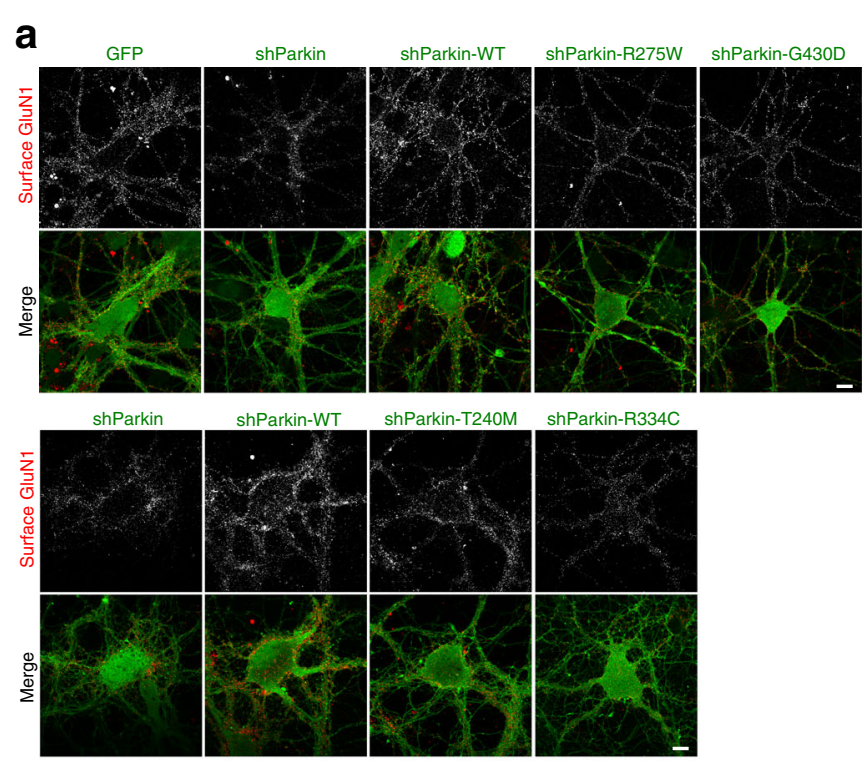

shParkin-T240M shParkin-R334C

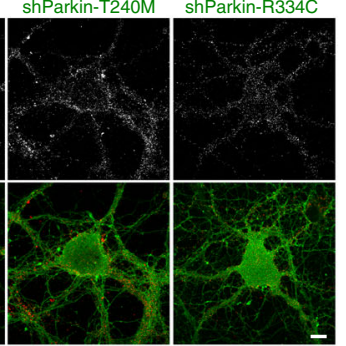

b
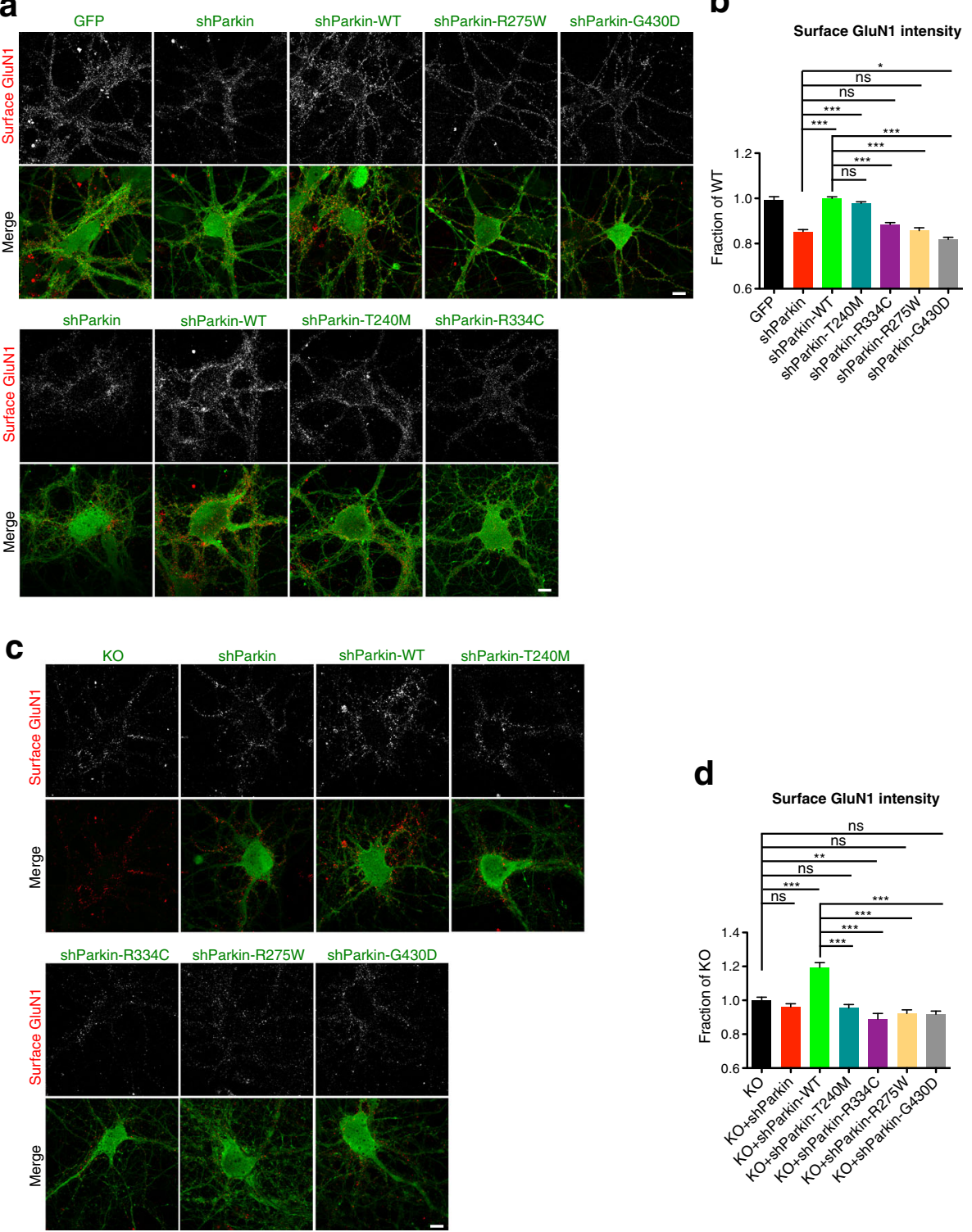

Fig. 2 Parkin mutation/loss-of-function decreases cell-surface GluN1 levels. a Representative images of surface GluN1 staining (red) in 14-16 DIV hippocampal neurons expressing GFP, shParkin, shParkin-WT, shParkin-T240M, shParkin-R275W, shParkin-R334C, or shParkin-G430D constructs. Scale bar, $10 \mu \mathrm{m}$. b Quantification of cell-surface GluN1 intensity expressed as a fraction of shParkin-WT ( $n \geq 50$ fields of view per condition with $>100$ GluN1 puncta per field, results confirmed in 4 independent experiments. ${ }^{* *} P<0.001$, one-way ANOVA, error bars represent SEM). Scale bar, $10 \mu \mathrm{m}$. c Representative images of surface GluN1 staining (red) in 14-16 DIV Parkin KO hippocampal neurons expressing shParkin, shParkin-WT, shParkin-T240M, shParkin-R275W, shParkin-R334C or shParkin-G430D constructs, and non-transduced KO control. Scale bar, $10 \mu \mathrm{m}$. d Quantification of cell-surface GluN1 intensity expressed as a fraction of Parkin KO ( $n \geq 50$ fields of view per condition with $>100$ GluA1 puncta per field, results confirmed in 2 independent experiments. ${ }^{* *} P<0.001$, one-way ANOVA, error bars represent SEM)

KO backgrounds (Fig. 3a, b, d, e), consistent with our previous findings [19] and results with cell-surface GluA1 immunostaining (Fig. 1). WT Parkin expression also increased mEPSC frequency in the $\mathrm{KO}$ but not knockdown background (Fig. 3a, c, d, f), as expected given our previous results showing that AMPAR MEPSC frequency was decreased in Parkin KO but not knockdown neurons [19]. In contrast, none of the Parkin mutants was able to increase mEPSC amplitude above knockdown or KO levels, and none rescued mEPSC frequency in $\mathrm{KO}$ neurons, although $\mathrm{T} 240 \mathrm{M}$ and $\mathrm{R} 275 \mathrm{~W}$ did have small but significant effects on frequency in the knockdown background (Fig. 3a-f).

We also examined spontaneous EPSC amplitudes for NMDARs (NMDAR-EPSCs) in both Parkin knockdown and $\mathrm{KO}$ neurons. Here, we observed that WT Parkin 


\section{a}

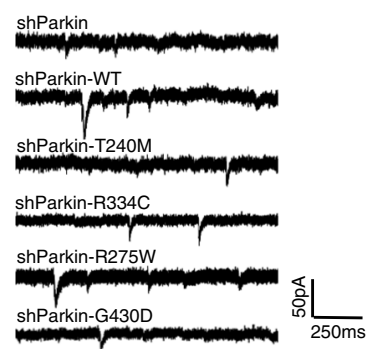

d

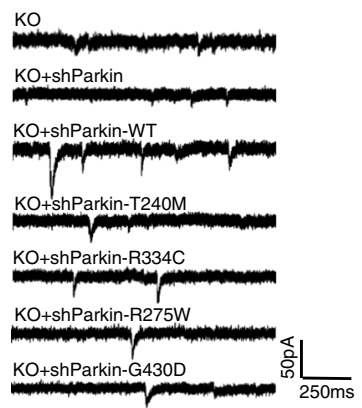

g

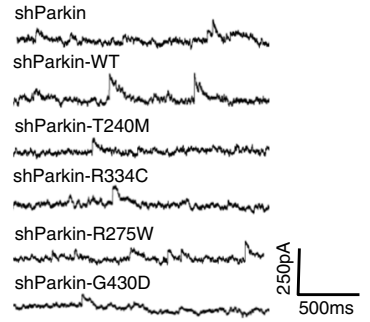

i

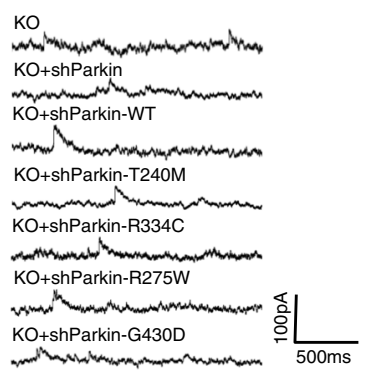

b

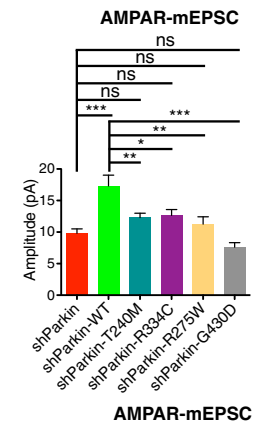

e

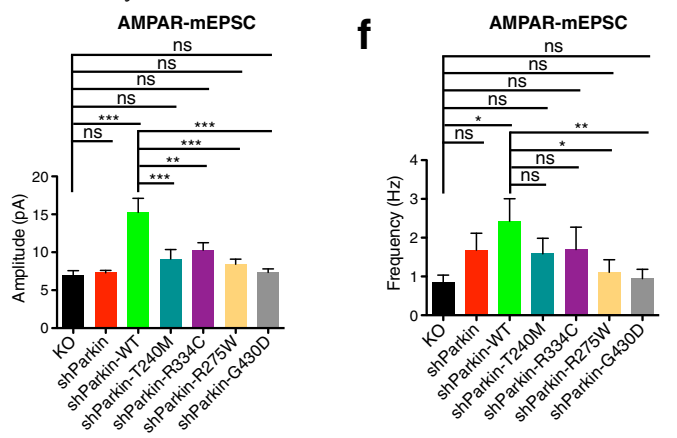

$\mathbf{h}$

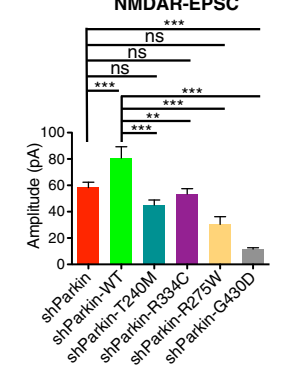

j

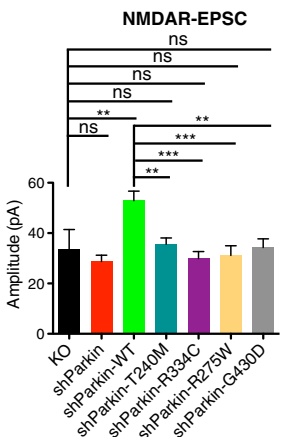

Fig. 3 (See legend on next page.) 


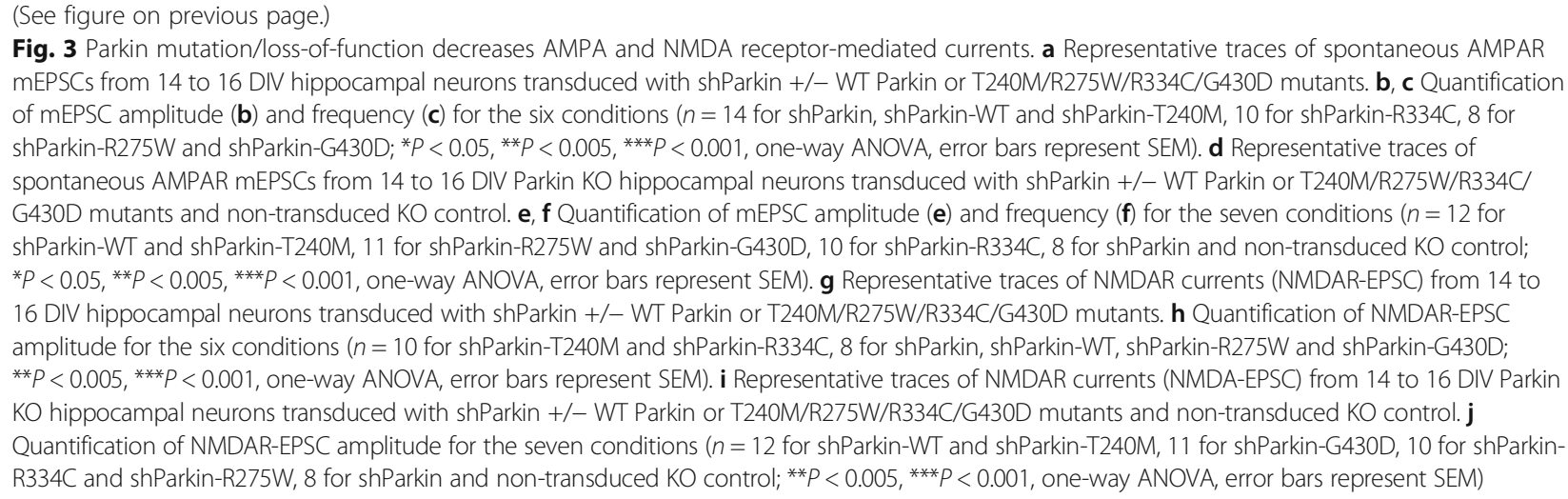

expression significantly increased NMDAR-EPSCs in both of these backgrounds (Fig. 3g-j), indicative of the reduction in synaptic NMDAR levels in the absence of Parkin. Again, the four Parkin mutants were unable to recapitulate WT Parkin function and did not increase NMDAR-EPSC amplitudes above knockdown/KO levels (Fig. 3g-j). To further confirm this loss of NMDARs in the Parkin-deficient background, we measured whole cell NMDAR currents in response to local application of NMDA for neurons expressing soluble GFP, human Parkin (Hu-Parkin), shParkin, or shParkin-WT (Additional file 2: Figure S2a), as well as the levels of NMDAR subunits (GluN1, GluN2A, and GluN2B) by cell-surface biotinylation (Additional file 2: Figure S2b and S2c). We observed that shParkin significantly reduced both NMDAR currents and surface GluN subunit biotinylation (Additional file 2: Figure S2), confirming by several methods that Parkin regulates cell-surface NMDAR levels.

\section{Parkin's E3 ligase activity is required for maintaining surface NMDAR but not AMPAR levels}

We next investigated the mechanisms through which these pathogenic mutations disrupt cell-surface AMPAR and NMDAR expression. Each mutant is reported or predicted to impair Parkin's E3 ligase activity [29, 30, 32], suggesting that the loss of Parkin-mediated ubiquitination leads to a reduction of cell-surface glutamate receptor levels. To test this, we took advantage of two mutants engineered to augment or inhibit Parkin-mediated ubiquitination. In particular, mutation of tryptophan 403 to alanine (W403A) was shown to increase Parkin's E3 ligase activity, and mutation of cysteine 431 to serine (C431S) to inhibit this activity [29](Additional file 1: Figure S1a). As in previous experiments, we expressed WT, W403A, or C431S Parkin together with shParkin in hippocampal neurons, and after verifying their expression (Additional file 1: Figure S1e), immunostained with GluN1 or GluA1 antibodies. Unexpectedly, we found that both W403A and C431S mutants efficiently restored surface GluA1 to WT
Parkin levels (Fig. 4a, c), while only W403A rescued surface GluN1 levels (Fig. 4b, d). These findings demonstrate that Parkin's E3 ligase activity is essential for maintaining cell-surface NMDAR but not AMPAR levels, and indicate that Parkin regulates each receptor type through a distinct mechanism. Further supporting this concept, we found that overexpression of the postsynaptic scaffold Homer1, previously shown to rescue surface GluA1 levels in Parkin-deficient neurons, did not rescue surface GluN1 levels (Additional file 3: Figure S3).

\section{Parkin mutants are deficient in ubiquitination of NMDAR subunit GluN1}

To determine whether Parkin regulation of surface NMDAR levels is mediated through direct ubiquitination of NMDAR subunits, we performed ubiquitination assays in HEK293T cells co-transfected with GFP-tagged NMDAR or AMPAR subunits (GluN1, GluN2B, GluA1, GluA2) together with HA-ubiquitin, Myc vector control or Myc-Parkin. Here, we found that Parkin co-expression significantly increased ubiquitination of GFP-GluN1 (Fig. 5a, b), and that a similar degree of GluN1 ubiquitination was observed following its immunoprecipitation under denaturing conditions (1\% SDS) to eliminate other potential binding partners (Additional file 4: Figure S4a, b). In contrast, the other glutamate receptor subunits had ubiquitination levels equivalent to that of GFP control (Fig. 5a, b). Follow-up co-immunoprecipitation assays revealed that GluN1 was also the only subunit to co-precipitate with Myc-Parkin (Fig. 5c). These data demonstrate that Parkin specifically interacts with and ubiquitinates the GluN1 subunit of NMDARs.

To evaluate whether the GFP-tagged Parkin mutants were impaired in their ability to ubiquitinate GluN1, we performed ubiquitination assays using Flag-GluN1. We first tested the sensitivity of the assay by examining GluN1 ubiquitination by the W403A (active) and C431S (inactive) Parkin mutants. Here, we found that W403A increased GluN1 ubiquitination compared to WT 


\section{a}

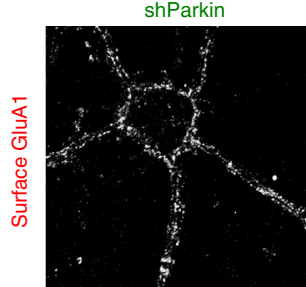

shParkin-WT

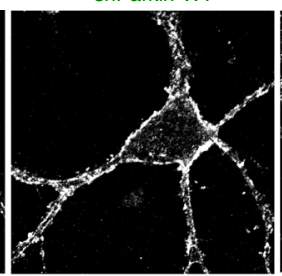

shParkin-C431S

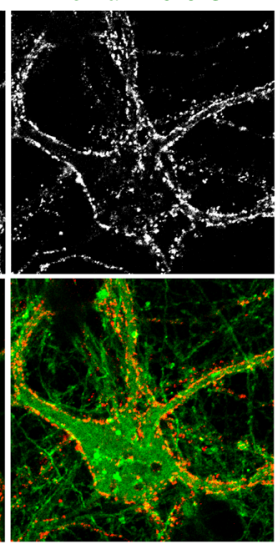

shParkin-W403A
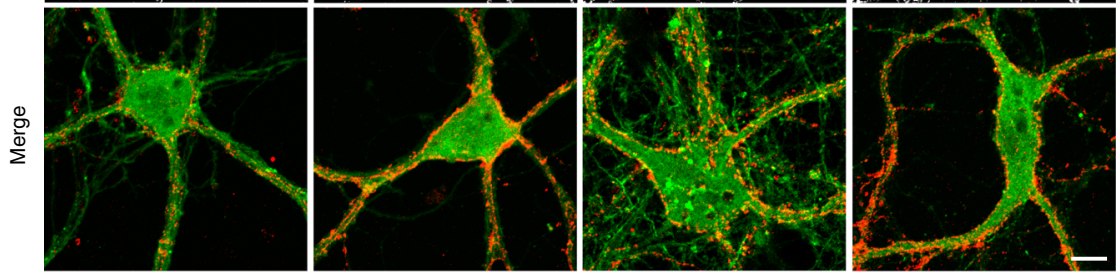

b

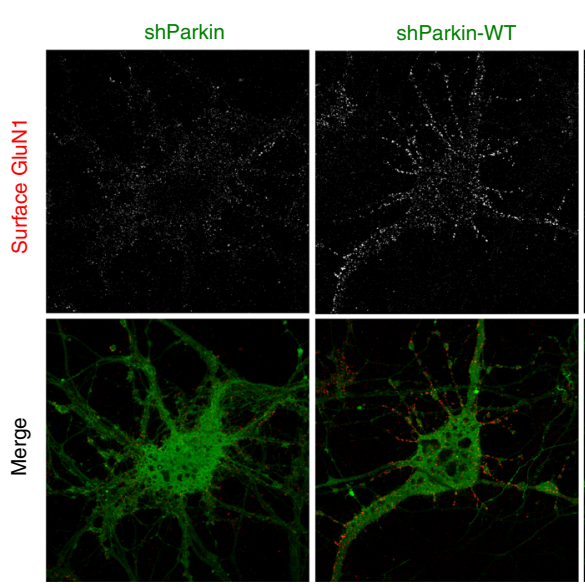

shParkin-C431S

shParkin-W403A

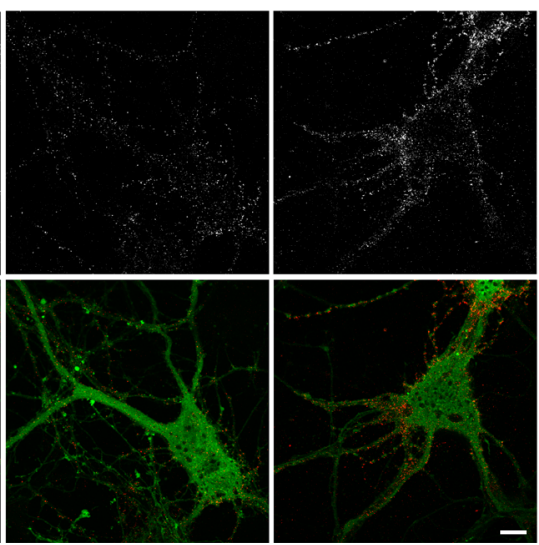

C

Surface GluA1 intensity

d

Surface GluN1 intensity
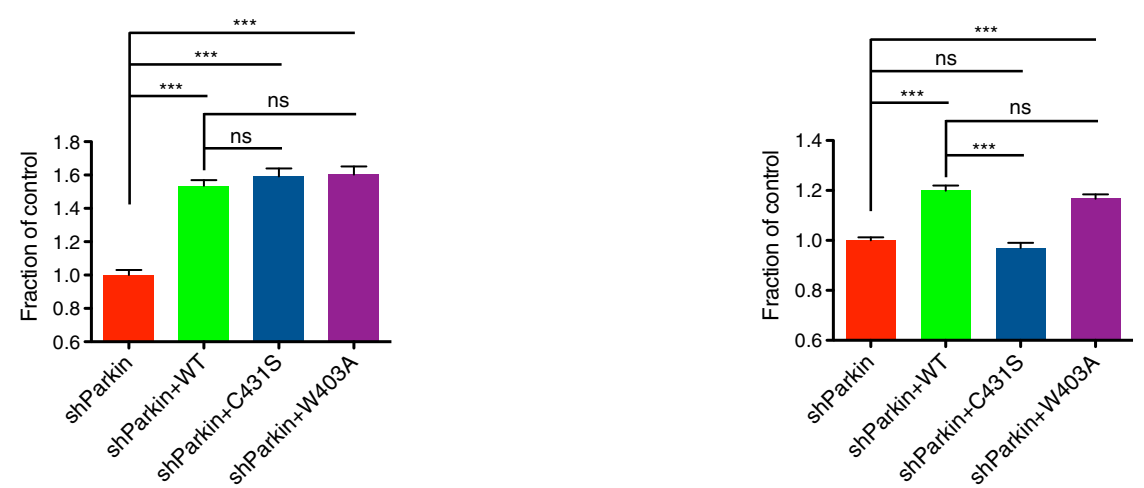

Fig. 4 Parkin E3 ligase activity is required to maintain cell-surface NMDARs but not AMPARs. a Representative images of surface GluA1 staining (red) in 14-16 DIV hippocampal neurons expressing shParkin +/- WT, C431S, or W403A Parkin constructs. Scale bar, 10 um. b Same condition as (a), but for surface GluN1 staining (red). Scale bar, $10 \mu \mathrm{m}$. c Quantification of cell- surface GluA1 intensity expressed as a fraction of shParkin control ( $n \geq 40$ fields of view per condition with $>100$ GluA1 puncta per field, results confirmed in 3 independent experiments. *** $P<0.001$, one-way ANOVA, error bars represent SEM). d Quantification of cell-surface GluN1 intensity expressed as a fraction of shParkin control ( $n \geq 40$ fields of view per condition with > 100 GluN1 puncta per field, results confirmed in 3 independent experiments. ${ }^{* * *} P<0.001$, one-way ANOVA, error bars represent SEM)

Parkin, while ubiquitination by C431S was indistinguishable from soluble GFP control (Fig. 5d, e). We next examined Flag-GluN1 ubiquitination in the presence of
T240M, R275W, R334C, and G430D and found the levels of ubiquitin immunoreactivity to be similar to those seen in the control condition (Fig. 5f, g), 
a

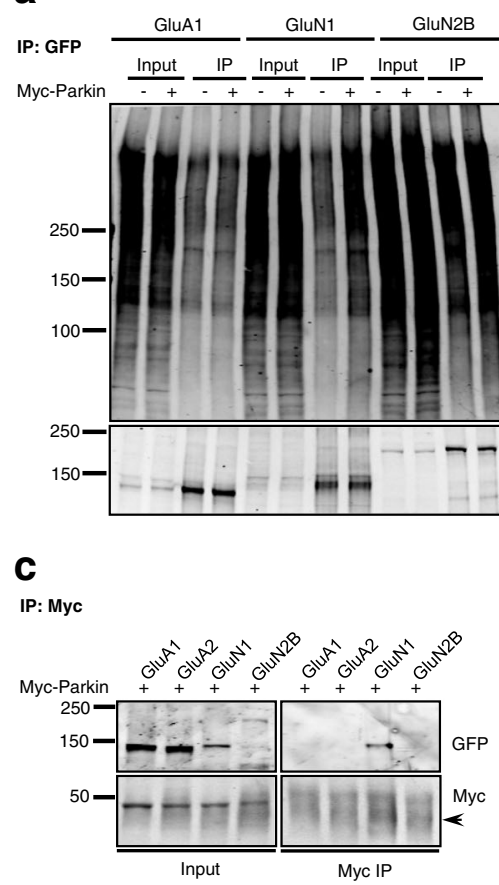

$\mathbf{f}$

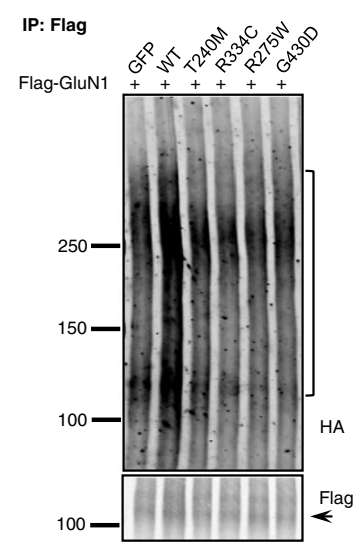

b

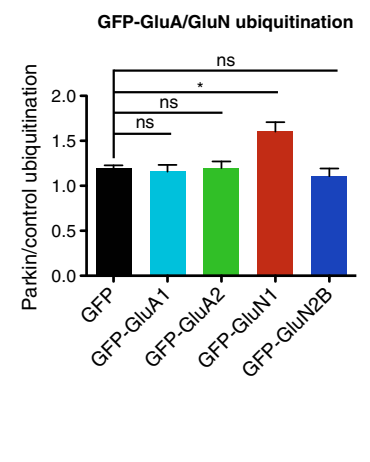

d

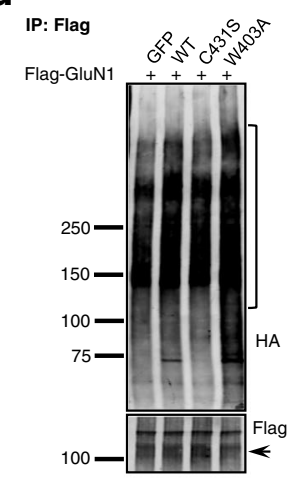

e

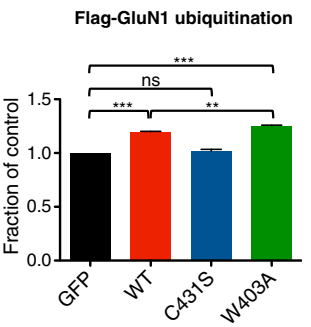

9 Flag-GluN1 ubiquitination

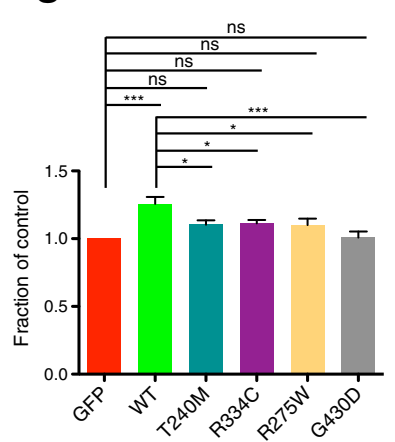

Fig. 5 Parkin-mediated GluN1 ubiquitination is impaired in pathogenic mutants. a Representative immunoblots for GFP immunoprecipitation (IP) from HEK293T cell lysates expressing Myc/Myc-Parkin, GFP-GluA1/-GluA2/-GluN1/-GluN2B, and HA-ubiquitin, probed for HA and GFP. Ubiquitin immunoreactivity used for quantification is marked on HA blots. b Quantification of GFP-GluA or GluN ubiquitination, expressed as the ratio of marked HA blot intensity (a) with Myc-Parkin (+) to Myc control (-), then normalized to immunoprecipitated GFP, GFP-GluA or GluN ( $n=3$ experiments, ${ }^{*} P<0.05$; one-way ANOVA, error bars represent SEM). c Representative Myc and GFP immunoblots for Myc IP from HEK293T cell lysates expressing Myc-Parkin and GFP-GluA1/-GluA2/-GluN1/-GluN2B. Arrowhead indicates immunoprecipitated Myc-Parkin (just below lgG band). d Representative HA and Flag immunoblots for Flag IP from HEK293T cell lysates expressing Flag-GluN1, GFP control/GFP-Parkin WT/ C431S/W403A, and HA-ubiquitin. Arrowhead indicates immunoprecipitated Flag-GluN1. Ubiquitin immunoreactivity used for quantification is marked on HA blots. e Quantification of Flag-GluN1 ubiquitination by measurement of marked HA blot intensity (d), normalized to immunoprecipitated Flag-GluN1 and reported as a fraction of GFP control $\left(n=3\right.$ experiments, ${ }^{*} P<0.01,{ }^{* *} P<0.001$, one-way ANOVA, error bars represent SEM). f Representative HA and Flag immunoblots for Flag IP from HEK293T cell lysates expressing Flag-GluN1, GFP control/GFP-Parkin WT/ T240M/R275W/R334C/G430D constructs, and HA-ubiquitin. Arrowhead indicates immunoprecipitated Flag-GluN1. Ubiquitin immunoreactivity used for quantification is marked on HA blots. $\mathbf{g}$ Quantification of Flag-GluN1 ubiquitination by measurement of marked HA intensity ( $\mathbf{f}$, normalized to immunoprecipitated Flag-GluN1 and reported as a fraction of GFP condition $\left(n=3\right.$ experiments, ${ }^{*} P<0.05$; ${ }^{* *} P<0.01$, *** $P<0.001$, one-way ANOVA, error bars represent SEM) 
confirming that these Parkin mutants are deficient in their ubiquitination of GluN1.

\section{Parkin regulates NMDAR internalization and recycling}

Ubiquitination is known to mediate protein degradation, and Parkin-mediated ubiquitination regulates the stability and degradation of multiple synaptic substrates [2124, 26]. However, since cell-surface GluN1 levels were decreased rather than increased following Parkin loss-of-function, we hypothesized that Parkin-mediated ubiquitination does not regulate NMDAR degradation. This hypothesis was confirmed by cycloheximide-chase experiments to compare the degradation of NMDAR subunits (GluN1, GluN2A, GluN2B) in control neurons with those expressing shParkin +/- WT Parkin (Additional file 4: Figure S4c, d). Since ubiquitination also regulates glutamate receptor trafficking [39-41], we utilized antibody feeding assays to monitor the internalization and recycling of GluN1 in neurons expressing soluble GFP or shParkin +/- WT-Parkin (see the "Methods" section for details). Here, we found that both the internalization of GluN1 and its recycling back to the cell-surface were significantly impaired in Parkin knockdown neurons and that these defects were fully rescued by WT Parkin (Fig. 6a-d). Altogether, these findings indicate that Parkin-mediated ubiquitination regulates both the endocytosis and exocytosis of NMDARs at the cell-surface, leading to an overall reduction of surface NMDARs in Parkin-deficient neurons.

\section{Parkin mutants are impaired in their binding and retention of postsynaptic Homer 1}

Although defective GluN1 ubiquitination provides a mechanistic explanation for how Parkin mutants decrease cell-surface NMDAR levels, it does not explain how these mutants decrease cell-surface AMPAR levels, a phenotype that does not appear to depend on Parkin's ubiquitinating activity (see Fig. 4). We previously showed that direct binding of Parkin to Homer1 is necessary for maintaining surface AMPAR levels, by tethering endocytic zones (EZs) for AMPAR capture and internalization to the postsynaptic density [19]. In Parkin-deficient neurons, Homer1 levels are decreased, leading to reduced EZ density and loss of synaptic AMPARs [19]. To determine whether the Parkin mutants were impaired in their binding to Homer1, we performed co-immunoprecipitation assays in HEK293T cells co-expressing GFP-tagged Homer1 and either T240M, R275W, R334C, or G430D. Intriguingly, all of the mutants exhibited significantly less co-precipitation with Homer1 than did WT Parkin (Fig. 7a, b), reinforcing the link between Parkin/Homer1 binding and surface GluA1 levels. We also performed quantitative immunofluorescence microscopy to measure synaptic Homer1 levels in knockdown neurons expressing WT Parkin or each mutant. Here, we found that synaptic Homer1 levels were significantly decreased in the presence of all four mutants compared to WT Parkin (Fig. 7c, d), consistent with our immunostaining and electrophysiology data showing decreased cell-surface AMPARs and mEPSC amplitudes (Figs. 1 and 3). Together, these findings provide an explanation for how Parkin mutations disrupt synaptic AMPAR levels and AMPAR-mediated currents.

\section{Parkin-mediated AMPAR internalization is required for induction of synaptic depression}

NMDAR-dependent synaptic plasticity mechanisms, including long-term potentiation (LTP) and long-term depression (LTD), are responsible for dynamic changes in synaptic strength that mediate important high-order brain functions such as learning and memory, pain perception, addictive behaviors, and mood regulation [42-44]. Induction of LTP and LTD require rapid, calcium-mediated changes in the number, phosphorylation state, and/or subunit composition of synaptic cell-surface AMPARs [45, 46]. Since we identified clear roles for Parkin in AMPAR and NMDAR trafficking, we hypothesized that Parkin mutation or loss-of-function would impair these synaptic plasticity mechanisms. Indeed, a previous study reported that Parkin KO animals exhibit reduced hippocampal LTP [20]. To test this possibility, we first examined NMDARdependent synaptic potentiation and depression in hippocampal neurons expressing soluble GFP or shParkin +/- WT Parkin, using chemical stimuli to induce LTP or LTD (cLTP or CLTD; see the "Methods" section and [47, 48]). Following these treatments, we calculated the ratio of mean surface GluA1 intensity following cLTP or cLTD induction to that in the untreated control condition, with values $>1$ representing insertion of GluA1-containing AMPARs at the cell surface (i.e., successful cLTP), and values $<1$ representing internalization of AMPARs from the surface (i.e., successful cLTD). Surprisingly, we found that the magnitude of cLTP was nearly identical for GFP control and shParkin-expressing neurons, although both the basal and potentiated levels of surface GluA1 were lower in knockdown neurons than in control neurons (Fig. 8a, b). On the other hand, co-expression of WT Parkin led to a slight impairment of cLTP induction, likely due to occlusion from higher initial surface levels of GluA1 (Fig. 8a, b). In contrast, Parkin knockdown completely prevented the induction of CLTD, and this phenotype was fully restored by WT Parkin expression (Fig. 8c, d). These data are consistent with our previous findings that Parkin regulates AMPAR endocytosis but not exocytosis [19].

We next evaluated whether co-expression of Parkin mutants could rescue this cLTD deficit. Remarkably, we found that none of the four mutants were able to rescue 


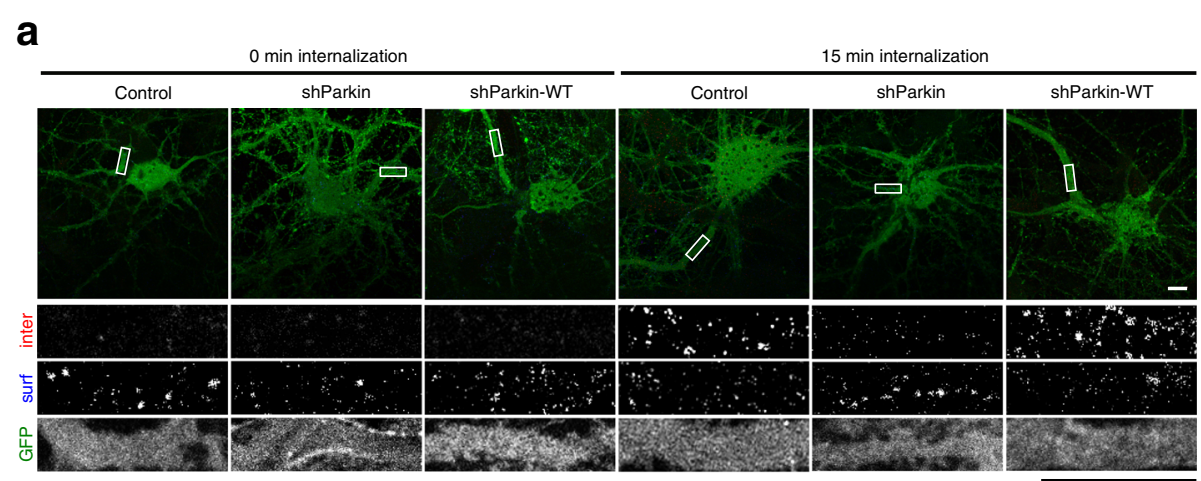

b

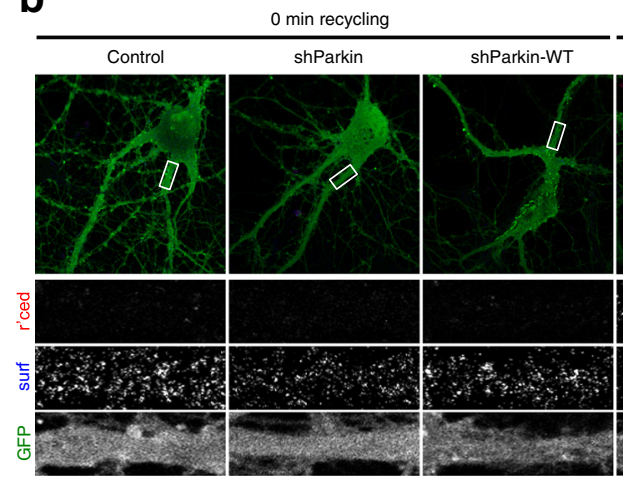

C
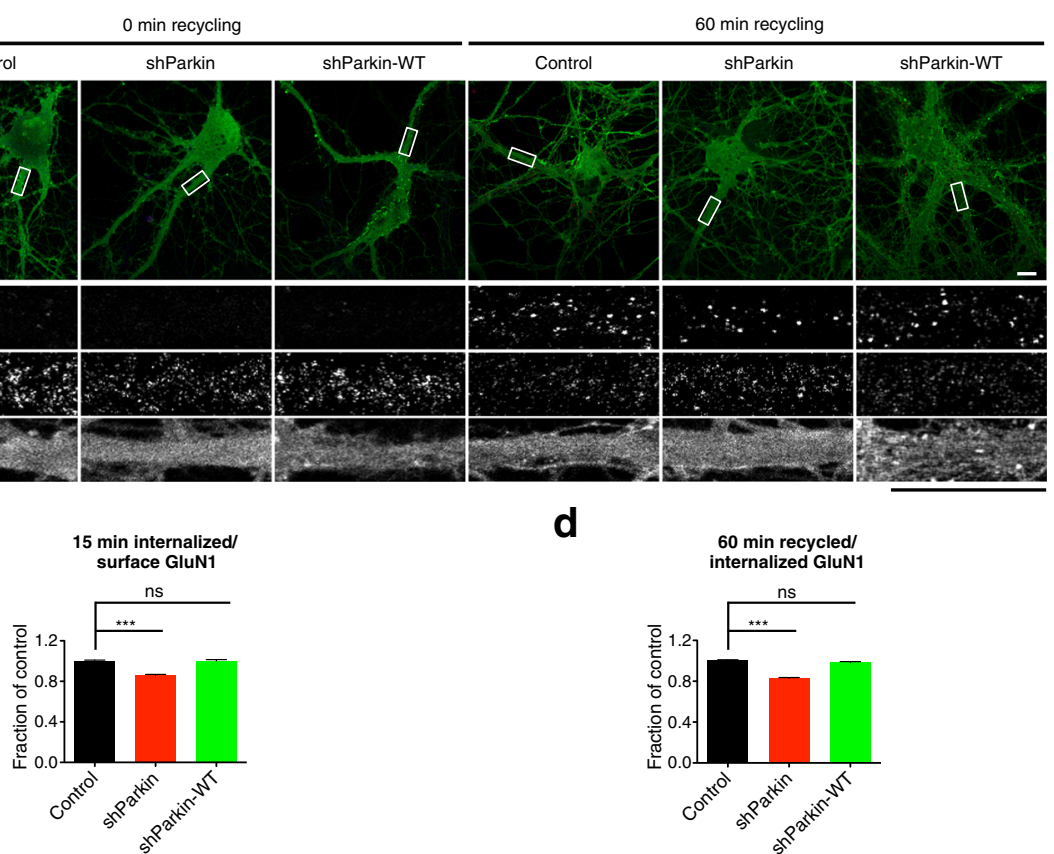

d

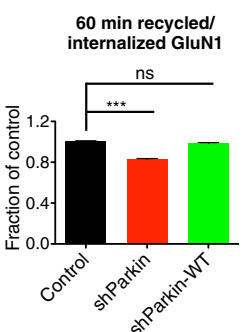

Fig. 6 Parkin deficiency impairs GluN1 internalization and recycling. a Representative images of 14-16 DIV hippocampal neurons expressing soluble GFP control/shParkin/shParkin-WT constructs, immunostained for cell surface (surf) and internalized (inter) GluN1 after 0 and 15 min internalization at $37^{\circ} \mathrm{C}$. Scale bars, $10 \mu \mathrm{m}$. b Representative images of 14-16 DIV hippocampal neurons expressing the same constructs as in (a), immunostained for cell surface (surf) and recycled (rec'd) GluN1 after 30 min internalization followed by 0 and 60 min recycling at $37^{\circ} \mathrm{C}$. Scale bars, $10 \mu \mathrm{m}$. c Quantification of GluN1 internalization at $15 \mathrm{~min}$, expressed as the ratio of internalized to cell surface GluN1 and normalized to GFP control condition ( $n \geq 50$ fields of view per condition with > 50 GluN1 puncta per field, results confirmed in 3 independent experiments; ${ }^{* *} P<0.001$, unpaired $t$ test, error bars represent SEM). d Quantification of GluN1 recycling at 60 min, expressed as the ratio of recycled to internalized GluN1 and normalized to GFP control condition ( $n \geq 55$ fields of view per condition with $>50$ GluN1 puncta per field, results confirmed in 3 independent experiments; ${ }^{* * *} P<0.001$, unpaired $t$ test, error bars represent SEM)

GluA1 internalization following cLTD induction (Fig. 8e, f), consistent with their reduced Homer1 binding and inability to rescue synaptic Homer1 levels compared to WT Parkin (Fig. 7). Supporting the concept that Parkin mutation/loss-of-function prevents LTD induction by disrupting Homer1-linked endocytic zones and thus GluA1 internalization, we found that dephosphorylation of GluA1 at Serine-845, a hallmark of LTD that is dependent upon events upstream of AMPAR internalization (i.e., $\mathrm{Ca}^{2+}$ influx through NMDARs, phosphatase activation; [47]), was unaffected by Parkin knockdown or mutant co-expression (Additional file 5: Figure S5). These findings indicate that pathogenic Parkin mutations disrupt synaptic depression by impairing AMPAR internalization.

Since hippocampal LTP was reported to be deficient in Parkin KO animals [20], we also examined cLTP induction in hippocampal neurons from the Parkin KO background, in the presence or absence of WT Parkin or the four mutants. We found that the magnitude of cLTP 


\section{a}

IP: Homer1

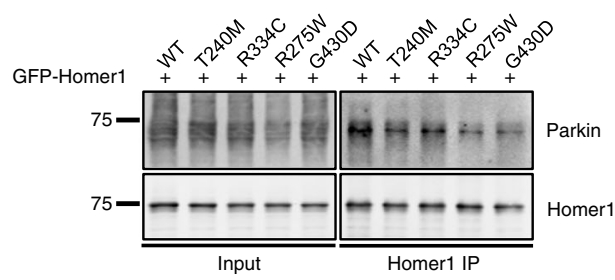

b

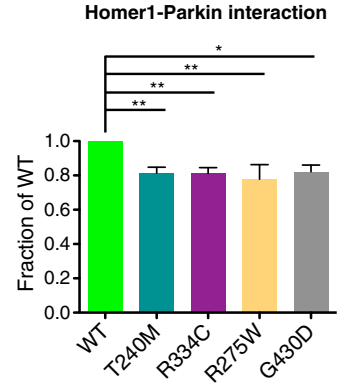

C

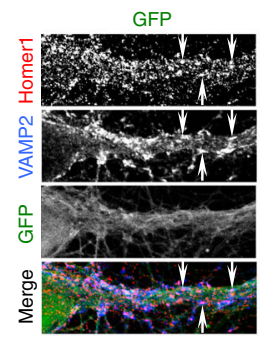

shParkin

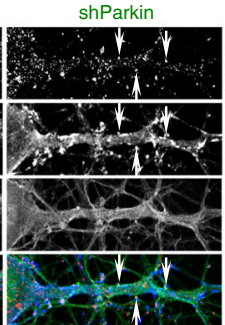

shParkin-WT

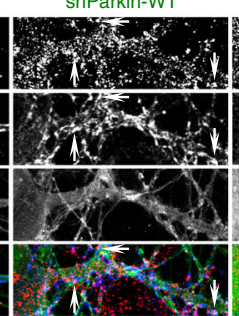

shParkin-R275W

shParkin-G430D

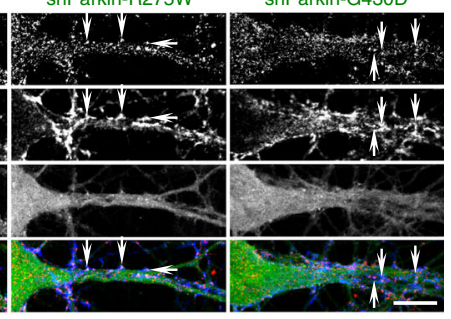

shParkin-R334C

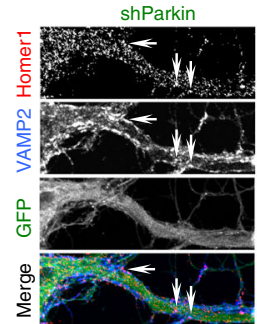

shParkin-WT

shParkin-T240M
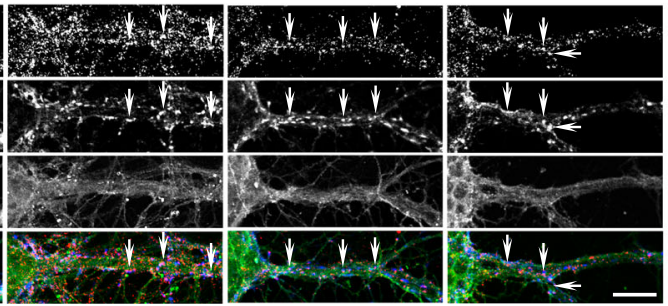

d

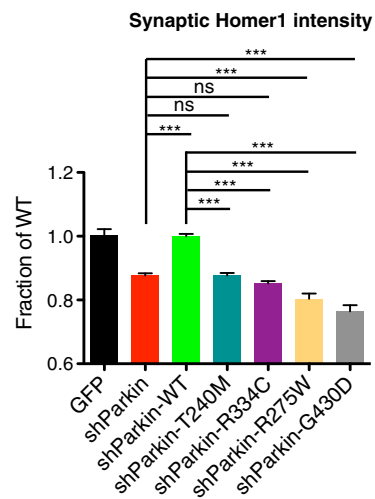

Fig. 7 Parkin mutations reduce Homer1 binding and synaptic retention. a Representative Parkin and Homer1 immunoblots for Homer1 IP from HEK293T cell lysates expressing GFP-Homer1 and GFP-Parkin WT/T240M/R275W/R334C/G430D. b Quantification of Homer1/Parkin interaction by measurement of co-immunoprecipitated GFP-Parkin WT/T240M/R275W/R334C/G430D, normalized to immunoprecipitated GFP-Homer1 and reported as a fraction of GFP-Parkin WT condition ( $n=3$ experiments, one-way ANOVA, ${ }^{*} P<0.05, * * P<0.01$, error bars represent SEM). c Representative images of Homer1 (red) and VAMP2 (blue) staining in 14-16 DIV hippocampal neurons expressing GFP, shParkin, or shParkin-WT/-T240M/-R275W/-R334C/-G430D. Arrows indicate representative synaptic Homer1 puncta (based on co-localization with VAMP2) for each condition. Scale bar, $10 \mu \mathrm{m}$. d Quantification of synaptic Homer1 intensity at VAMP2-immunopositive puncta along dendrites, expressed as a fraction of shParkin-WT control condition ( $n \geq 50$ fields of view per condition with $>50$ co-localized Homer 1 puncta per field, results confirmed in 3 independent experiments; ${ }^{* * *} P<0.001$, one-way ANOVA, error bars represent SEM)

induction as measured by surface GluA1 levels was indistinguishable in $\mathrm{KO}$ neurons compared to those expressing WT or mutant Parkin (Fig. 9a, b), again demonstrating that Parkin loss-of-function does not impair
LTP. However, induction of cLTD was completely blocked in $\mathrm{KO}$ neurons, and this phenotype was rescued only by WT Parkin expression (Fig. 9c, d), indicating that the four mutants cannot support cLTD in either the 

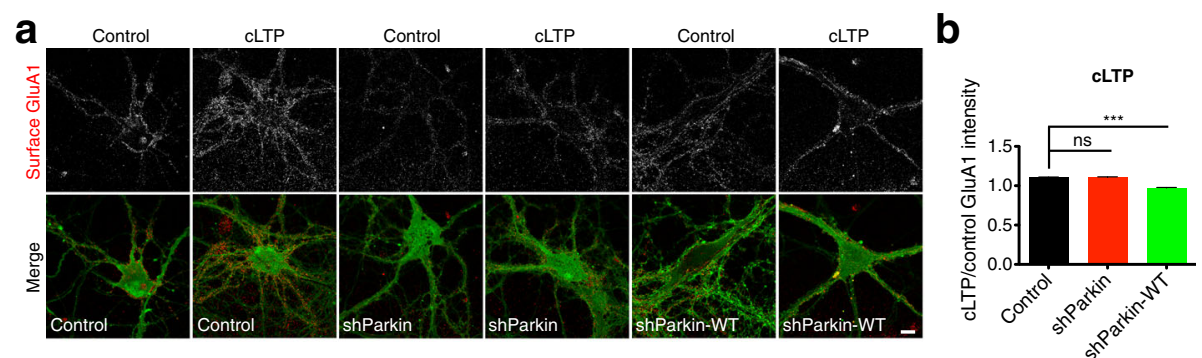

C Control

Control

CLTD

Control

CLTD
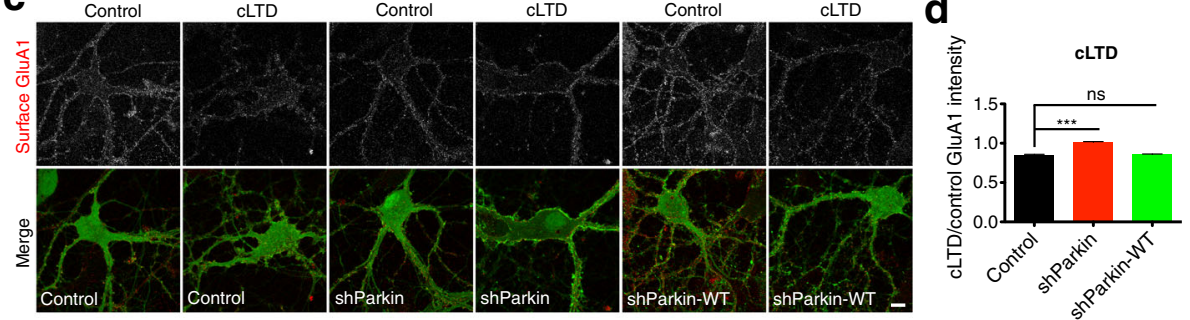

e
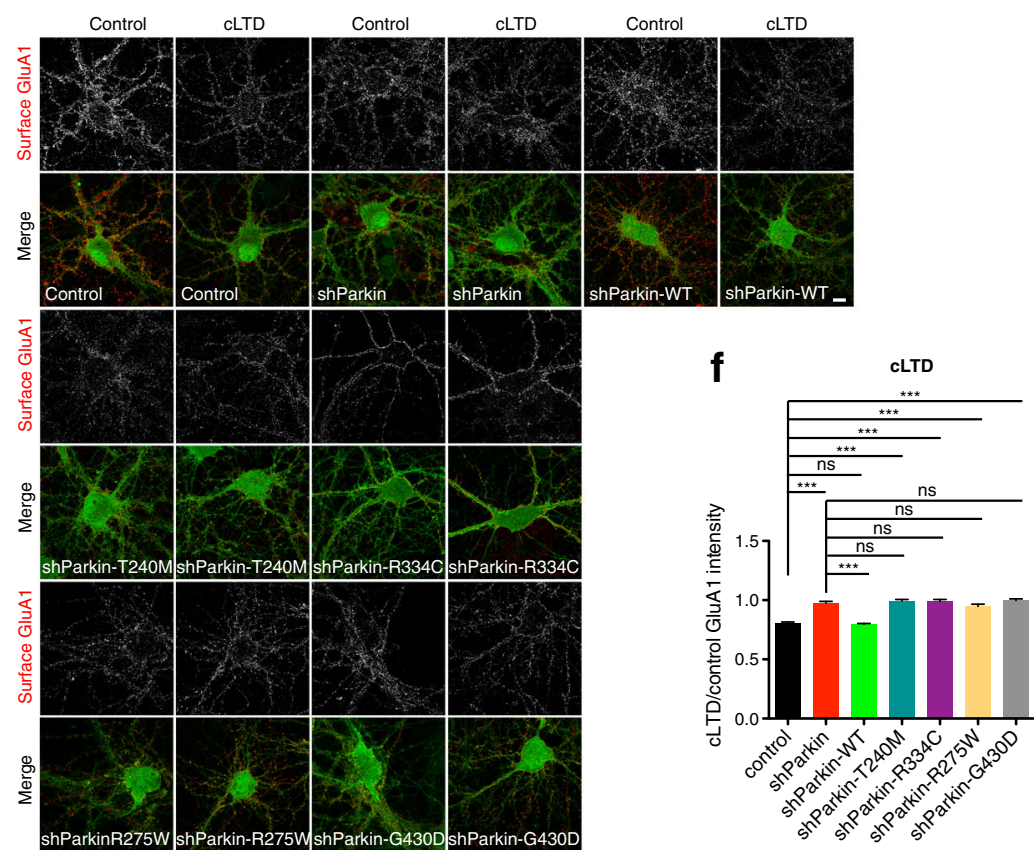

Fig. 8 Parkin mutation/knockdown impairs the induction of LTD. a Representative images of surface GluA1 staining (red) in 14-16 DIV hippocampal neurons expressing GFP, shParkin or shParkin-WT, under the control condition (no treatment) or after chemical LTP (cLTP) induction. Scale bar, $10 \mu \mathrm{m}$. b Quantification of the ratio of GluA1 intensity after CLTP induction to the control condition for neurons expressing GFP, shParkin, or shParkin-WT. c Same as (a), but for control condition or chemical LTD (CLTD) induction. Scale bar, $10 \mu \mathrm{m}$. d Quantification of the ratio of GluA1 intensity after CLTD induction to the control condition for neurons expressing GFP, shParkin, or shParkin-WT. e Representative images of surface GluA1 staining (red) in 14-16 DIV hippocampal neurons expressing GFP, shParkin or shParkin-WT/-T240M/-R275W/-R334C/-G430D constructs, under the control condition or after CLTD induction. Scale bar, $10 \mu \mathrm{m}$. $\mathbf{f}$ Quantification of the ratio of GluA1 intensity after CLTD induction to the control condition for neurons expressing GFP, shParkin or shParkin-WT/-T240M/-R275W/-R334C/-G430D constructs. For panels (b) and (d), $n \geq 50$ fields of view per condition with $>100 \mathrm{GluA1}$ puncta per field, results confirmed in 3 independent experiments. ${ }^{* * *} P<0.001$, unpaired $t$ test. For panel (f), $n \geq 40$ fields of view per condition with $>100$ GluA1 puncta per field, results confirmed in 3 independent experiments. ${ }^{* * *} P<0.001$, one-way ANOVA, error bars represent SEM

Parkin-deficient or Parkin-null backgrounds. Together, these findings indicate that Parkin mediates NMDARdependent synaptic depression by facilitating AMPAR internalization and that pathogenic Parkin mutations prevent this function.

\section{Discussion}

PARK2 mutations are present in $>3 \%$ of PD patients, making them more prevalent than mutations in LRRK2 or $\alpha$-synuclein [36, 37, 49-51]. Moreover, PD is a multisystem disorder and Parkin is enriched at postsynaptic 


\section{a}
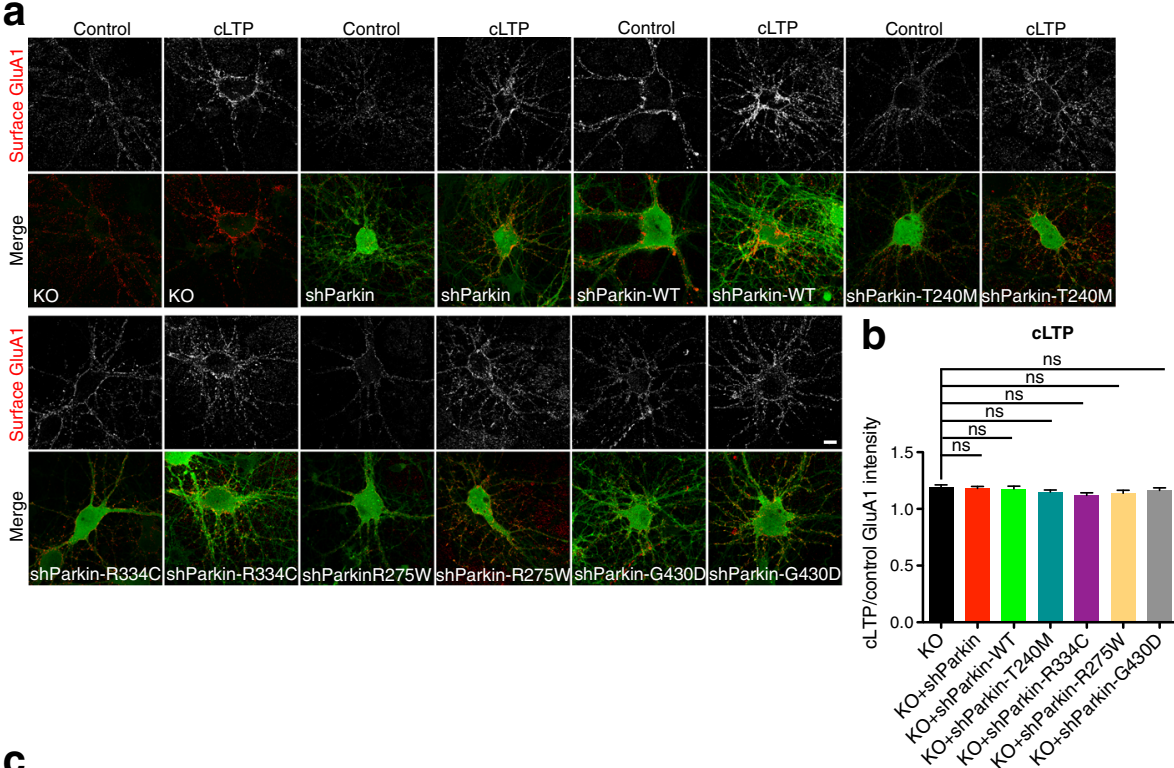

C
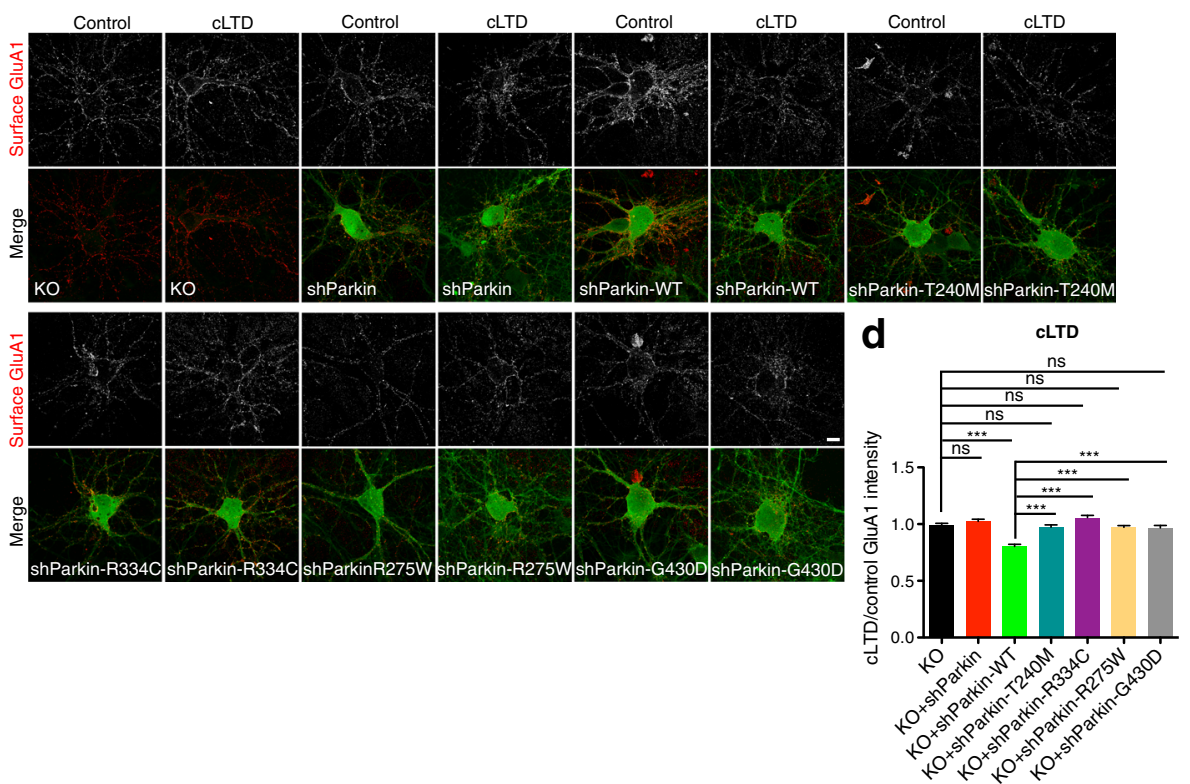

Fig. 9 Parkin mutation/knockout impairs the induction of LTD but not LTP. a Representative images of surface GluA1 staining (red) in 14-16 DIV Parkin KO hippocampal neurons expressing shParkin or shParkin-WT/-T240M/-R275W/-R334C/-G430D constructs, and non-transduced KO control under the control condition (no treatment) or after chemical LTP (CLTP) induction. Scale bar, $10 \mu m$. b Quantification of the ratio of GluA1 intensity after CLTP induction to the control condition for neurons expressing the above Parkin constructs. c Same as (a), but for control condition or chemical LTD (CLTD) induction. Scale bar, $10 \mu \mathrm{m}$. $\mathbf{d}$ Quantification of the ratio of GluA1 intensity after CLTD induction to the control condition for neurons expressing above Parkin constructs. For panels (b) and (d), $n \geq 40$ fields of view per condition with > 100 GluA1 puncta per field, results confirmed in 2 independent experiments. ${ }^{* *} P<0.001$, one-way ANOVA, error bars represent SEM

densities (PSDs) of glutamatergic synapses, suggesting that PARK2 mutations could contribute to PD pathophysiology by disrupting excitatory neurotransmission. Here, we demonstrate that expression of four PD-linked Parkin mutations (T240M, R275W, R334C, and G430D) in Parkin-deficient or Parkin-null backgrounds, to simulate its heterozygous or homozygous loss-of-function, alters glutamatergic synaptic transmission and plasticity. We provide mechanistic insight into how these Parkin mutations impair NMDAR and AMPAR-mediated signaling, through deficient ubiquitination of GluN1 and deficient binding/retention of postsynaptic Homer1, leading to reduced cell-surface NMDAR and AMPAR levels and impaired AMPAR internalization. Our 
findings not only demonstrate that Parkin regulates postsynaptic NMDA- and AMPA-type glutamate receptors through distinct mechanisms, but also that common PD-linked mutations disrupt both functions. Furthermore, these studies show that even partial loss of Parkin function significantly alters excitatory neurotransmission and plasticity, suggesting that heterozygous PARK2 mutations also impair these processes.

Although multiple studies show that Parkin regulates glutamatergic neurotransmission, it is currently unclear how its loss-of-function impacts excitatory drive in the brain. Several studies report that Parkin mutation/ loss-of-function increases AMPAR and kainate receptor (KAR)-mediated currents in neurons, enhances glutamate excitotoxicity, and increases KAR and NMDAR levels in several brain regions [14, 17, 26, 52]. These findings have led to the hypothesis that Parkin loss-of-function increases postsynaptic glutamate receptor levels and currents, promoting the death of dopaminergic neurons by excitotoxicity [14, 52]. However, other groups report that Parkin mutation/loss-of-function decreases neuronal excitability and/or AMPAR EPSCs, as well as AMPAR levels in brain tissue from knockout mice and human patients $[15,16,19,26,52$, 53]. We similarly find that Parkin loss-of-function decreases AMPAR- and NMDAR-mediated currents and cell-surface levels, suggesting that pathogenic Parkin mutations may reduce basal excitatory drive. On the other hand, our finding that Parkin deficiency/loss-of-function prevents the induction of LTD in hippocampal neurons, potentially leading to an inability to weaken or depotentiate glutamatergic synapses, lends support to the concept of increased excitation or altered excitatory/ inhibitory balance in brains lacking Parkin.

Hippocampal NMDAR-dependent LTP is reportedly impaired in Parkin KO mice [20], and we find that cell-surface NMDAR levels are significantly reduced in neurons lacking enzymatically active Parkin due to its knockdown/KO or mutation, suggesting that synaptic plasticity mechanisms could be broadly disrupted. However, we find that LTP induction is intact in hippocampal neurons lacking functional Parkin, but that LTD is impaired. These findings suggest that the decrease in surface NMDARs does not disrupt all NMDAR-dependent plasticity mechanisms, although it may lead to more subtle deficits in LTP that we cannot detect in our analyses. These findings are also consistent with our previous work showing that Parkin is not required for AMPAR exocytosis, but is an important regulator of AMPAR endocytosis through its stabilization of Homer1-linked endocytic zones [19]. Interestingly, none of the four Parkin mutants evaluated in this study were able to rescue either postsynaptic Homer1 levels or LTD induction, and all exhibited significantly reduced Homer1 binding capacity.
These findings suggest that Parkin's ability to mediate AMPAR internalization during LTD induction is intertwined with its ability to interact with and maintain Homer1 at the PSD.

PARK2-linked PD is often considered a distinct disease from idiopathic or other genetic forms of PD, with earlier onset, slower progression, and more purely motoric symptoms [7, 54]. Consistent with these clinical phenotypes, PARK2 patient brains lack widespread pathology, including the Lewy bodies found in other forms of PD, and cell loss is restricted to dopaminergic neurons of the substantia nigra [7]. Predominantly glutamatergic brain regions, including the hippocampus, are not primarily affected or implicated in this form of PD. However, PARK2 patients and heterozygous PARK2 mutation carriers are reported to exhibit higher rates of neuropsychiatric symptoms (e.g., depression, psychosis, panic attacks) than non-carriers $[7,54,55]$, and there is a significant association between PARK2 heterozygosity and obsessivecompulsive disorder [56]. While these disorders are associated with altered dopaminergic signaling, they are also strongly linked to changes in glutamatergic neurotransmission, including decreased AMPAR and NMDAR levels in specific brain regions [57-60]. PARK2 patients also exhibit unusual sensitivity to L-dopa treatment and rapid development of L-dopa-induced dyskinesia. Interestingly, dyskinesia in animal models and PD patients is linked to impaired glutamatergic synaptic plasticity, and in particular to the inability of synapses to become depotentiated/ depressed following L-dopa-induced potentiation [61-63]. Our findings that Parkin-deficient excitatory synapses can become potentiated but not depressed, and that pathogenic Parkin mutations similarly do not support the induction of NMDAR-dependent LTD, may provide an explanation for the development of dyskinesia in PARK2 patients. Furthermore, increased potentiation of excitatory synapses would lead to higher levels of cell-surface AMPARs, potentially hastening the death of alreadyvulnerable dopaminergic neurons by glutamate excitotoxicity and thereby contributing to the early onset of motor symptoms in PARK2 patients. It is also worth noting that recent genome-wide association studies link PARK2 deletions and copy number variations to autism, schizophrenia, and intellectual disability [33, 64-66], disorders commonly associated with dysfunction of glutamatergic synapses. Such findings highlight the need for further investigation of Parkin's role in excitatory neurotransmission, and in particular how disruption of glutamatergic signaling due to its loss-of-function may contribute to the pathophysiology of PD and other brain disorders.

\section{Conclusions}

Together, our studies implicate glutamatergic synaptic dysfunction in the etiology of motor and non-motor 
symptoms experienced by PARK2 patients. Moreover, given the recent link between $P A R K 2$ copy number variation and neurodevelopmental disorders, our findings further underscore the importance of elucidating Parkin's molecular mechanisms of action at glutamatergic synapses, both to facilitate the development of better treatments for motor and non-motor symptoms of PARK2-linked PD, and to shed light on its emerging role in the etiology of neuropsychiatric disease.

\section{Methods}

\section{Antibodies and reagents}

The following primary antibodies and dilutions were used for western blot and immunoprecipitation: mouse Parkin (Prk8, 1:1000; Santa Cruz Biotechnology), GluN1 (1:500; EMD Millipore), GluN2A (1:500; EMD Millipore), GluN2B (1:500; Neuromab), GluA1 phospho-Serine 845 rabbit antibody (1:1,000; Cell Signaling Technology), mouse tubulin (1:10,000; Sigma), rabbit tubulin (1:10,000; Abcam), rabbit GFP (1:1000; Invitrogen), mouse Myc (1:500; Santa Cruz Biotechnology), mouse HA (1:500; Santa Cruz Biotechnology), and mouse Flag M2 (1:5,000; Sigma). DyLight fluorescent secondary antibodies were purchased from Thermo Fisher Scientific and diluted 1:15,000. The following primary antibodies and dilutions were used for immunostaining: purified human antiGluN1 antiserum (a kind gift from C. Garner, German Center for Neurodegenerative Diseases (DZNE)) with $1 \mu \mathrm{g} / \mathrm{ml}$ working concentration, mouse GluA1 (1:100; EMD Millipore), rabbit Homer1 (1:500; Synaptic System), unconjugated anti-mouse/human secondary antibody (1:100; Invitrogen), mouse VAMP2 (1:500; Synaptic System), and Alexa Fluor 568/647 anti-mouse/human secondary antibody (1:400; Invitrogen). Pharmacological agents used are as follows: cycloheximide (Calbiochem, $0.05 \mu \mathrm{g} / \mu \mathrm{l}, 24 \mathrm{~h}$ ), NMDA, AP5, CNQX, and TTX (Tocris Bioscience), bicuculline, strychnine, leupeptin $(50 \mu \mathrm{M})$, chloroquine $(50 \mu \mathrm{M})$ and epoxomicin $(0.1 \mu \mathrm{M})$ (Sigma), and PR-619 (LifeSensors, $50 \mu \mathrm{M}$ ). Unless otherwise indicated, all other chemicals are from Sigma-Aldrich.

\section{Primary hippocampal culture}

Rat primary hippocampal cultures were prepared using a modified Banker culture protocol (Banker and Goslin, 1998; Waites et al., 2009). Briefly, neurons from embryonic (E18-E19) Sprague Dawley rat hippocampi, taken from animals of both sexes, were dissociated in TrypLE Express (ThermoFisher) for $20 \mathrm{~min}$, washed with HBSS (Sigma), and plated in Neurobasal medium with B27 supplement and Glutamax (all from ThermoFisher) at a density of 250,000 neurons per well (12-well plates) or coverslip $(22 \times 22 \mathrm{~mm}$ square $)$.

\section{Plasmids and transfection/transduction}

The target sequence of shParkin and subcloning of shParkin +/-human Parkin constructs into pFUGW H1 have been described previously [19]. The following plasmids were purchased from Addgene: pCI-SEP-GluA1/ -GluA2/-GluN1/-GluN2B (all from Dr. Robert Malinow), pEGFP-Parkin C431S/W403A (from Dr. Edward Fon), pRK5-Myc-Parkin, and pRK5-HA-ubiquitin (from Dr. Ted Dawson). Parkin mutants (T240M, R275W, R334C, G430D) were synthesized from Genewiz and subcloned into pEGFP-C2 and pFUGW H1-shParkin vector. pEGFP-Parkin C431S/W403A were also subcloned into FUGW H1-shParkin vector. Flag-GluN1 construct was a gift from $\mathrm{O}$. Jeyifous (University of Chicago). The GFP-Homer1 construct was a gift from A. M. Grabrucker (University of Ulm, Ulm, Germany) and C. Garner. pFUGW constructs were used to generate lentivirus for transduction of primary neurons as described previously [67], except that Calfectin (SignaGen Laboratories) was used for transfection of HEK293T cells. HEK medium was replaced with Neurobasal medium 18-24 h after transfection, and this medium (viral supernatant) was harvested $24 \mathrm{~h}$ later. Neurons were transduced with $50-100 \mu \mathrm{l}$ of lentiviral supernatant per well at $2-3$ days in vitro (DIV) and used for experiments between 13 and 15 DIV. This time course was optimized based on efficacy of the shRNA knockdown. Transfections were performed on 5 DIV using Lipofectamine 2000 (Invitrogen) as described previously [19].

\section{Electrophysiology}

Whole-cell patch-clamp recordings were performed in hippocampal neurons plated onto astrocyte microislands or 12-mm coverslips and transduced with the indicated constructs, as previously described [19]. Cultures were perfused at room temperature with an extracellular solution $(145 \mathrm{mM} \mathrm{NaCl}, 5 \mathrm{mM} \mathrm{KCl}, 10 \mathrm{mM}$ HEPES, $1.3 \mathrm{mM}$ $\mathrm{MgCl}_{2}, 2 \mathrm{mM} \mathrm{CaCl} 2,10 \mathrm{mM}$ glucose) containing $1 \mu \mathrm{M}$ of TTX (mEPSCs only) and $100 \mu \mathrm{M}$ picrotoxin. Electrodes had resistances between 3 and $7 \mathrm{M} \Omega$ containing intracellular solution $(110 \mathrm{~mm}$ Cs-methanesulfonate, $10 \mathrm{mM}$ Na-methanesulfonate, $10 \mathrm{mM}$ EGTA, $1 \mathrm{mM} \mathrm{CaCl}$, $10 \mathrm{mM}$ HEPES, $10 \mathrm{mM}$ TEA, $5 \mathrm{mM}$ QX-314, $5 \mathrm{mM}$ MgATP, $0.5 \mathrm{mM}$ NaGTP). Neurons were visualized by fluorescence microscopy, and those expressing GFP were selected for recordings. For AMPA receptor current events, neurons were held at $-65 \mathrm{mV}$ and mEPSCs were recorded over 5 min using a MultiClamp 700B amplifier (Molecular Devices) controlled with a PC running MultiClamp Commander and pClamp (Molecular Devices) and pass filtered at $2 \mathrm{kHz}$. For miniature NMDA currents (NMDAR-EPSCs), neurons were voltage clamped at + $40 \mathrm{mV}$ and recorded over $5 \mathrm{~min}$. For pharmacological induction of NMDARs, currents were measured following 
local application of NMDA (100 $\mu \mathrm{M}$; Tocris Bioscience), using a custom-built application system $\sim 200 \mu \mathrm{m}$ from synapsing neurons on a specific microisland, in addition to bath application of the AMPAR antagonist CNQX (10 $\mu \mathrm{M}$; Tocris Bioscience) while holding the neuron at + $40 \mathrm{mV}$. Data acquisition and offline analysis of EPSCs were performed with pClamp (Clampex version 10.4). Approximately 15-20 neurons were analyzed for each experimental condition from three to four independent cultures.

\section{Immunoblotting}

Cultured neurons were collected directly in $\times 2$ SDS sample buffer (Bio-Rad). Samples were subjected to SDS-PAGE, transferred to nitrocellulose membranes, probed with primary antibodies in 5\% BSA/PBS plus $0.05 \%$ Tween 20 overnight at $4{ }^{\circ} \mathrm{C}$ or $1 \mathrm{~h}$ at room temperature, followed by Dylight fluorescent secondary antibodies for $1 \mathrm{~h}$. Membranes were imaged using an Odyssey Infrared Imager (model 9120; LI-COR Biosciences). Protein bands were quantified using the ImageJ (NIH) "Gels" function, and all bands were normalized to their loading controls.

\section{Biotinylation assay}

Cultured cortical neurons transduced with GFP/shParkin/ shParkin-WT (2-14 DIV) were washed with ice-cold PBS containing $1 \mathrm{mM} \mathrm{MgCl} 2$ and $0.1 \mathrm{mM} \mathrm{CaCl}_{2}$ (PBS+) and incubated with $1 \mathrm{mg} / \mathrm{ml}$ EZ-Link Sulfo-NHS-LC-biotin (ThermoFisher) in PBS+ for $20 \mathrm{~min}$ at $4{ }^{\circ} \mathrm{C}$ with gentle agitation. Cells were washed with ice-cold quenching buffer (50 mM glycine in PBS+) for $10 \mathrm{~min}$ at $4{ }^{\circ} \mathrm{C}$, lysed in ice cold lysis buffer $50 \mathrm{mM}$ Tris-base, $150 \mathrm{mM} \mathrm{NaCl}, 2 \%$ Triton X-100, 0.5\% deoxycholic acid, and supernatant was incubated with streptavidin-Sepharose beads (ThermoFisher) for $2 \mathrm{~h}$ at $4{ }^{\circ} \mathrm{C}$. Bound proteins were immunoblotted with GluN1, GluN2A, GluN2B, Parkin, and tubulin. The data were quantified by measuring surface receptor to total input receptor band intensity ratios using ImageJ software and normalizing to GFP control cultures.

\section{Co-immunoprecipitation assay}

For co-immunoprecipitation studies, HEK293T cells were transfected with plasmids (pCI-SEP-GluA1/-GluA2/ -GluN1/-GluN2B and Myc-Parkin for Myc-IP assay; GFP-Homer1 and GFP control/GFP-Parkin WT/T240M/ R275W/R334C/G430D for Homer1 IP assay) using Calfectin according to the manufacturer's (SignaGen Laboratories) protocol. Cell lysates were collected $36 \mathrm{~h}$ after transfection in lysis buffer (50 mM Tris-base, $150 \mathrm{mM}$ $\mathrm{NaCl}, 1 \%$ Triton X-100, 0.5\% deoxycholic acid) with protease inhibitor mixture (Roche) and clarified by centrifugation at high speed $(20,000 \mathrm{rcf})$. The resulting supernatant was incubated with Dynabeads (ThermoFisher) coupled with anti-Myc or anti-Homer1 antibodies at $4{ }^{\circ} \mathrm{C}$ under constant rotation for $2-4 \mathrm{~h}$. Beads were washed two to three times with lysis buffer and once with PBS. Bound proteins were eluted using sample buffer (Bio-Rad) and subject to SDS-PAGE immunoblotting.

\section{Ubiquitination assay}

HEK293T cells were transfected with pCI-SEP-GluA1/ -GluA2/-GluN1/-GluN2B, Myc-Parkin and HA-ubiquitin, or with GFP/GFP-Parkin WT/-Parkin C431S/-Parkin W403A/-Parkin T240M/-Parkin R275W/-Parkin R334C/ -Parkin G430D, Flag-GluN1, and HA-Ub using Calfectin according to the manufacturer's protocol. Thirty-six hours after transfection, proteasome and lysosome inhibitors including leupeptin, chloroquine, epoxomicin, and PR619 were added to the cultures. After 2-4-h treatment, cell lysates were collected in lysis buffer with protease inhibitor mixture and clarified by centrifugation at high speed $(20,000 \mathrm{rcf})$. The resulting supernatant was incubated with Dynabeads (ThermoFisher) coupled with anti-GFP antibodies or anti-Flag M2 magnetic beads (Sigma) at $4{ }^{\circ} \mathrm{C}$ under constant rotation for overnight. Beads were washed two to three times with lysis buffer and once with PBS. Bound proteins were eluted using sample buffer (Bio-Rad) and subject to SDS-PAGE immunoblotting.

\section{Immunofluorescence microscopy}

Primary antibodies and concentrations are listed above. Alexa Fluor 488-, Alexa Fluor 568-, or Alexa Fluor 647-conjugated secondary antibodies (ThermoFisher) were used at 1:400. Neurons were immunostained as described previously [67]. Briefly, coverslips were fixed with Lorene's fixative $(60 \mathrm{mM}$ PIPES, $25 \mathrm{mM}$ HEPES, $10 \mathrm{mM}$ EGTA, $2 \mathrm{mM} \mathrm{MgCl} 2,0.12 \mathrm{M}$ sucrose, $4 \%$ formaldehyde) for $15 \mathrm{~min}$, primary and secondary antibody incubations were performed in blocking buffer ( $2 \%$ glycine, $2 \% \mathrm{BSA}, 0.2 \%$ gelatin, and $50 \mathrm{mM} \mathrm{NH} 4 \mathrm{Cl}$ in $\times 1$ PBS) for $1-2 \mathrm{~h}$ at room temperature or overnight at $4^{\circ}$ $\mathrm{C}$, and all washes were done with PBS. For surface labeling, GluN1 $(1 \mu \mathrm{g} / \mathrm{ml})$ or GluA1 antibody (1:100) was added to live neurons, incubated for $30 \mathrm{~min}$ at room temperature, washed three times in PBS, and fixed and stained with secondary antibodies as described. Coverslips were mounted with VectaShield (Vector Laboratories) and sealed with clear nail polish. Images were acquired with a $\times 63$ objective (Neofluar, NA 1.4) on a Zeiss LSM 800 confocal microscope running Zen2 software.

\section{GluN1 internalization assay}

GluN1 internalization was measured using a protocol as described previously [19]. Neurons expressing GFP/ shParkin/shParkin-WT were incubated with human GluN1 antibody ( $1 \mu \mathrm{g} / \mathrm{ml}$ in PBS) for $30 \mathrm{~min}$ at room temperature to label surface NMDARs, washed four 
times with PBS, and either fixed immediately with Lorene's fixative or incubated at $37{ }^{\circ} \mathrm{C}$ for 15 min before fixation to allow for receptor internalization. Cells were then incubated with anti-human Alexa Fluor $647 \mathrm{sec}-$ ondary antibody diluted in blocking buffer (used for all remaining steps) for $40 \mathrm{~min}$ at room temperature, washed three times, and incubated for $30 \mathrm{~min}$ with unconjugated anti-mouse secondary (1:100) antibody to block any remaining unlabeled cell-surface GluN1 antibody. Finally, cells were washed four times, permeabilized with $0.25 \%$ Triton X-100, and incubated for $1 \mathrm{~h}$ at room temperature with anti-human Alexa Fluor 568 to label internalized GluN1.

\section{GluN1 recycling assay}

Neurons were incubated with anti-GluN1 antibody to label surface receptors as described above and incubated at $37^{\circ} \mathrm{C}$ for $30 \mathrm{~min}$ to allow for receptor internalization. All non-internalized surface antibody was subsequently blocked with an unconjugated anti-human secondary antibody at room temperature for $30 \mathrm{~min}$ and washed four times in PBS. Next, neurons were fixed or incubated at $37{ }^{\circ} \mathrm{C}$ for $1 \mathrm{~h}$ to allow for receptor recycling back to the plasma membrane. They were then fixed, incubated for $40 \mathrm{~min}$ with Alexa Fluor 568-conjugated anti-mouse secondary antibody diluted in blocking buffer (used for all remaining steps) to label the recycled surface population of receptors, and washed three times. The remaining surface primary antibodies were blocked with an excess of an unconjugated anti-human secondary antibody at room temperature for $30 \mathrm{~min}$, and neurons again were washed four times. After permeabilization, neurons were incubated with Alexa Fluor 647-conjugated anti-human secondary antibody for $40 \mathrm{~min}$ to label the internalized population of receptors, washed three times, and mounted and imaged as described previously.

\section{Chemical LTP/LTD assay}

Chemical LTP/LTD assay was performed as described previously [68]. Neurons were washed twice in Tyrodes solution $(120 \mathrm{mM} \mathrm{NaCl}, 2 \mathrm{mM} \mathrm{CaCl}, 2.5 \mathrm{mM} \mathrm{KCl}$, $25 \mathrm{mM}$ HEPES, $30 \mathrm{mM}$ glucose, $\mathrm{pH}$ 7.4), and then incubated in cLTP buffer $(200 \mu \mathrm{M}$ glycine, $1 \mu \mathrm{M}$ strychnine, $20 \mu \mathrm{M}$ bicuculline in Tyrodes solution, $\mathrm{pH}$ 7.4) for $5 \mathrm{~min}$. Following a quick wash with wash buffer $(2 \mathrm{mM}$ $\mathrm{MgCl}_{2}, 50 \mu \mathrm{M}$ AP5, $10 \mu \mathrm{M}$ CNQX, $0.5 \mu \mathrm{M}$ TTX, $1 \mu \mathrm{M}$ strychnine in Tyrodes solution, $\mathrm{pH}$ 7.4), neurons were incubated for another $5 \mathrm{~min}$ in this buffer prior to fixation. For chemical LTD, neurons were washed twice in Tyrodes solution, then incubated with cLTD buffer (25 $\mu \mathrm{M}$ NMDA, $1 \mu \mathrm{M}$ strychnine, $0.5 \mu \mathrm{M}$ TTX, $10 \mu \mathrm{M}$ glycine in Tyrodes solution, $\mathrm{pH}$ 7.4) for 5 min followed by a quick wash with wash buffer. After cLTP or cLTD treatment, neurons were subjected to surface GluA1 staining for $10 \mathrm{~min}$, then fixed and immunostained with secondary antibody.

\section{Image analyses}

Images of surface or total GluN1/GluA1 were manually thresholded to select only puncta that were greater than twofold above image background. The average intensity values of puncta were measured using the "Analyze Particles" function in Image//Fiji, with puncta size set between 2 and 100 pixels. Intensity of Homer1 puncta at sites of VAMP2 colocalization was calculated by creating a selection in ImageJ/Fiji using the VAMP2 channel, applying it to the relevant Homerl channel, and measuring average intensity values of Homer1 puncta using the Analyze Particles function, with puncta size set between 2 and 100 pixels. For internalization and recycling assays, ratios of internalized GluN1 intensity to surface GluN1 intensity, or recycled to internalized GluN1 intensity, were calculated per punctum (after measuring raw intensity values in the red and far-red channels) and averaged for each field of view. These averaged ratios were then normalized to the control condition (average value set to 1) and expressed as a fraction of control.

\section{Statistical analysis}

Statistical analyses were performed in GraphPad Prism5 software using either one-way ANOVA-Tukey, ANOVADunnett's multiple comparison test, or unpaired, two-tailed Student's $t$ tests, with $P<0.05$ considered significant. Data are presented as mean \pm SEM.

\section{Additional files}

Additional file 1: Figure S1. Expression of Parkin mutants in hippocampal neurons. (a) Schematic diagram of Parkin showing domains and mutation/ deletion sites. (b) Immunoblots of lysates from 14 DIV hippocampal neurons expressing GFP control, shParkin, shParkin-WT, shParkin-T240M, shParkinR275W, shParkin-R334C, or shParkin-G430D constructs, probed for Parkin, GFP, and tubulin. (c) Representative images of shParkin-WT, shParkin-T240M, shParkin-R334C, shParkin-R275W and shParkin-G430D expression in 14 DIV hippocampal neurons. Scale bar, 10 um. (d) Immunoblots of lysates from 14 DIV Parkin KO hippocampal neurons expressing shParkin, shParkin-WT, shParkin-T240M, shParkin-R275W, shParkin-R334C or shParkin-G430D constructs and Parkin KO control, probed for Parkin, GFP, and tubulin. (e) Immunoblots of lysates from hippocampal neurons expressing GFP, shParkin, shParkin-WT, shParkin-C431S, or shParkin-W403A, probed for Parkin, GFP, and tubulin. Note that GFP-Parkin W403A is not recognized by the Parkin antibody but is detected by the GFP antibody. (PDF $3931 \mathrm{~kb}$ )

Additional file 2: Figure S2. Parkin deficiency leads to reduced NMDAR-mediated currents and cell-surface levels. (a) Representative traces of whole-cell currents (upper panel) induced by local application of $100 \mu \mathrm{M}$ NMDA to hippocampal neurons expressing GFP, human Parkin (Hu-Parkin), shParkin, or shParkin-WT constructs, and quantification of peak current amplitudes induced by NMDAR activation for these conditions (lower panel) ( $n=19$ for control, 24 for shParkin, 22 for hu-Parkin, 14 for rescue; ${ }^{*} P<0.05$, ${ }^{* *} P<0.005$, one-way ANOVA, error bars represent SEM). (b) Representative immunoblots of total input (left) and surface biotin-labeled fractions (right) from 14 DIV hippocampal neuron lysates expressing GFP, shParkin or shParkin-WT constructs. (c) Quantification of surface/input ratio, 
normalized to GFP control condition and expressed as a fraction of control. $\left(n=3\right.$ experiments; ${ }^{*} P<0.05 ;{ }^{* *} P<0.01,{ }^{* * *} P<0.001$, unpaired $t$ test, error bars represent SEM). (PDF $215 \mathrm{~kb}$ )

Additional file 3: Figure S3. Homer1 overexpression does not rescue cell-surface NMDAR levels in Parkin knockdown neurons. (a) Representative images of hippocampal neurons co-transfected from 6 to 14 DIV with mCherry + /- shParkin alone or with GFP-Homer1, and immunostained for surface GluN1. Scale bar, $10 \mu \mathrm{m}$. (b) Quantification of cell surface GluN1, expressed as a fraction of mCherry control $(n \geq 40$ fields of view per condition with $>100$ GluN1 puncta per field, results confirmed in 3 independent experiments. ${ }^{* *} P<0.001$, one-way ANOVA, error bars represent SEM). (PDF $5670 \mathrm{~kb}$ )

Additional file 4: Figure S4. Parkin-mediated ubiquitination does not induce NMDAR degradation. (a) Representative immunoblots for GFP immunoprecipitation (IP) under denaturing conditions (1\% SDS) from HEK293T cell lysates expressing Myc/Myc-Parkin, GFP-GluN1 and HAubiquitin, probed for HA and GFP. Ubiquitin immunoreactivity used for quantification is marked on HA blots. (b) Quantification of GFP-GluN1 ubiquitination by measurement of marked HA blot intensity, normalized to immunoprecipitated GFP-GluN1 and reported as a fraction of Myc control. ( $n=3$ experiments, ${ }^{*} P<0.05$; one-way ANOVA, error bars represent SEM). (c) Representative immunoblots of lysates from 14 DIV hippocampal neurons expressing GFP/shParkin/shParkin-WT constructs, treated with DMSO vehicle control (-) or cycloheximide (CHX) and probed with the indicated antibodies. (d) Quantification of protein intensity after $24 \mathrm{~h}$ of $\mathrm{CHX}$ treatment, normalized to tubulin and reported as a fraction of the DMSO control intensity (starting material) $(n=3$ experiments, unpaired $t$ test, error bars represent SEM). (PDF $178 \mathrm{~kb}$ )

Additional file 5: Figure S5. Parkin has no effect on phospho-Serine 845 GluA1 reduction during CLTD. (a) Representative immunoblots of lysates from 14 DIV hippocampal neurons expressing GFP/shParkin/sh Parkin-WT/-T240M/-R334C constructs, under control condition (-) or after CLTD induction (+) and probed with the indicated antibodies. (b) Quantification of the ratio of phospho-Serine 845 GluA1 intensity with CLTD to control condition, normalized to GFP control. ( $n=3$ experiments, one-way ANOVA, error bars represent SEM). (PDF $119 \mathrm{~kb}$ )

\section{Abbreviations}

AMPA: a-Amino-3-hydroxy-5-methyl-4-isoxazolepropionic acid; GluA: AMPA receptor; GluN: NMDA receptor; NMDA: N-Methyl-D-aspartic acid; PD: Parkinson's disease

\section{Acknowledgements}

We thank Dr. Craig Garner (German Center for Neurodegenerative Diseases (DZNE), Universitätsmedizin Berlin) for the generous gift of human GluN1 antibody, Drs. Okinola Jeyifous and William Green (University of Chicago) for pFlag-GluN1 construct, Dr. Robert Malinow for pCI-SEP-GluA1/-GluA2/GluN1/-GluN2B constructs (Addgene plasmids \#23997-24001), Dr. Edward Fon for pEGFP-Parkin WT/C431S/W403A constructs (Addgene plasmids \#45875-77), Dr. Ted Dawson for pRK5-Myc-Parkin and pRK5-HA-Ubiquitin-WT constructs (Addgene plasmids \#17612 and \#17608, respectively), and Dr. A.M. Grabrucker (University of Ulm, Germany) for GFP-Homer1 construct. We would also like to thank Dr. Damian Williams (CUMC electrophysiology core) for technical help with electrophysiology experiments, and Cyndel Vollmer for help with glial microisland preparation.

\section{Funding}

This work was supported by NIH/NINDS grant number NS080967, and Brain Research Foundation Fay/Frank Seed Grant to C.W.

\section{Availability of data and materials}

All data analyzed in this study are included within the article.

\section{Authors' contributions}

MZ, GPC, and CLW designed the research; GPC did the electrophysiology experiments and analyses; MZ performed all other experiments and data analyses; MZ and CLW wrote the manuscript. All authors read and approved the final manuscript.

\section{Ethics approval and consent to participate}

Animal experimental procedures were reviewed and approved by the Institutional Animal Care and Use Committee of Columbia University.

\section{Consent for publication}

All authors have given consent for publication.

\section{Competing interests}

The authors declare that they have no competing interests.

\section{Publisher's Note}

Springer Nature remains neutral with regard to jurisdictional claims in published maps and institutional affiliations.

\section{Author details}

${ }^{1}$ Department of Pathology and Cell Biology, Columbia University Medical Center, New York, NY 10032, USA. ²Department of Psychiatry, Columbia University Medical Center, New York, NY, USA. ${ }^{3}$ Department of Neuroscience, Columbia University, New York, NY, USA. "Waites Lab, 650 W. 168th St. Black Building 1210B, New York, NY 10032, USA.

Received: 19 July 2018 Accepted: 24 August 2018

Published online: 10 September 2018

References

1. Kitada T, Asakawa S, Hattori N, Matsumine H, Yamamura Y, Minoshima S, et al. Mutations in the parkin gene cause autosomal recessive juvenile parkinsonism. Nature. 1998;392(6676):605-8.

2. Lucking CB, Durr A, Bonifati V, Vaughan J, De Michele G, Gasser T, et al. Association between early-onset Parkinson's disease and mutations in the Parkin gene. N Engl J Med. 2000;342(21):1560-7.

3. Periquet M, Latouche M, Lohmann E, Rawal N, De Michele G, Ricard S, et al. Parkin mutations are frequent in patients with isolated early-onset parkinsonism. Brain. 2003;126(Pt 6):1271-8.

4. Mata IF, Lockhart PJ, Farrer MJ. Parkin genetics: one model for Parkinson's disease. Hum Mol Genet 2004;13 Spec No 1:R127-R133.

5. Charan RA, LaVoie MJ. Pathologic and therapeutic implications for the cell biology of parkin. Mol Cell Neurosci. 2015;66(Pt A):62-71.

6. Zhang CW, Hang L, Yao TP, Lim KL. Parkin regulation and neurodegenerative disorders. Front Aging Neurosci. 2015;7:248

7. Hattori N, Mizuno Y. Twenty years since the discovery of the parkin gene. J Neural Transm (Vienna). 2017;124(9):1037-54

8. Geisler S, Holmstrom KM, Skujat D, Fiesel FC, Rothfuss OC, Kahle PJ, et al. PINK1/Parkin-mediated mitophagy is dependent on VDAC1 and p62/ SQSTM1. Nat Cell Biol. 2010;12(2):119-31.

9. Narendra DP, Jin SM, Tanaka A, Suen DF, Gautier CA, Shen J, et al. PINK1 is selectively stabilized on impaired mitochondria to activate Parkin. PLoS Biol. 2010;8(1):e1000298.

10. Vives-Bauza C, Zhou C, Huang Y, Cui M, de Vries RL, Kim J, et al. PINK1dependent recruitment of Parkin to mitochondria in mitophagy. Proc Nat Acad Sci U S A. 2010:107(1):378-83.

11. Matsuda N, Sato S, Shiba K, Okatsu K, Saisho K, Gautier CA, et al. PINK1 stabilized by mitochondrial depolarization recruits Parkin to damaged mitochondria and activates latent Parkin for mitophagy. J Cell Biol. 2010; 189(2):211-21.

12. Valadas JS, Vos M, Verstreken P. Therapeutic strategies in Parkinson's disease: what we have learned from animal models. Ann N Y Acad Sci. 2015:1338: $16-37$.

13. Morais VA, Verstreken $P$, Roethig A, Smet J, Snellinx A, Vanbrabant M, et al. Parkinson's disease mutations in PINK1 result in decreased complex I activity and deficient synaptic function. EMBO Mol Med. 2009;1(2):99-111.

14. Sassone J, Serratto G, Valtorta F, Silani V, Passafaro M, Ciammola A. The synaptic function of parkin. Brain. 2017;140(9):2265-72.

15. Goldberg MS, Fleming SM, Palacino JJ, Cepeda C, Lam HA, Bhatnagar A, et al. Parkin-deficient mice exhibit nigrostriatal deficits but not loss of dopaminergic neurons. J Biol Chem. 2003;278(44):43628-35.

16. Itier JM, Ibanez P, Mena MA, Abbas N, Cohen-Salmon C, Bohme GA, et al. Parkin gene inactivation alters behaviour and dopamine neurotransmission in the mouse. Hum Mol Genet. 2003;12(18):2277-91. 
17. Helton TD, Otsuka T, Lee MC, Mu Y, Ehlers MD. Pruning and loss of excitatory synapses by the parkin ubiquitin ligase. Proc Natl Acad Sci U S A. 2008;105(49):19492-7.

18. Kitada T, Pisani A, Karouani M, Haburcak M, Martella G, Tscherter A, et al. Impaired dopamine release and synaptic plasticity in the striatum of parkin -/- mice. J Neurochem. 2009;110(2):613-21.

19. Cortese GP, Zhu M, Williams D, Heath S, Waites CL. Parkin deficiency reduces hippocampal Glutamatergic neurotransmission by impairing AMPA receptor endocytosis. J Neurosci. 2016;36(48):12243-58.

20. Rial D, Castro AA, Machado N, Garcao P, Goncalves FQ, Silva HB, et al. Behavioral phenotyping of Parkin-deficient mice: looking for early preclinical features of Parkinson's disease. PLoS One. 2014;9(12):e114216.

21. Zhang Y, Gao J, Chung KK, Huang H, Dawson VL, Dawson TM. Parkin functions as an E2-dependent ubiquitin- protein ligase and promotes the degradation of the synaptic vesicle-associated protein, CDCrel-1. Proc Natl Acad Sci U S A. 2000;97(24):13354-9.

22. Huynh DP, Scoles DR, Nguyen D, Pulst SM. The autosomal recessive juvenile Parkinson disease gene product, parkin, interacts with and ubiquitinates synaptotagmin XI. Hum Mol Genet. 2003;12(20):2587-97.

23. Chung KK, Zhang Y, Lim KL, Tanaka Y, Huang H, Gao J, et al. Parkin ubiquitinates the alpha-synuclein-interacting protein, synphilin-1: implications for Lewy-body formation in Parkinson disease. Nat Med. 2001;7(10):1144-50.

24. Kabayama H, Tokushige N, Takeuchi M, Kabayama M, Fukuda M, Mikoshiba K. Parkin promotes proteasomal degradation of synaptotagmin IV by accelerating polyubiquitination. Mol Cell Neurosci. 2017;80:89-99.

25. Joch M, Ase AR, Chen CX, MacDonald PA, Kontogiannea M, Corera AT, et al. Parkin-mediated monoubiquitination of the PDZ protein PICK1 regulates the activity of acid-sensing ion channels. Mol Biol Cell. 2007;18(8):3105-18.

26. Maraschi A, Ciammola A, Folci A, Sassone F, Ronzitti G, Cappelletti G, et al. Parkin regulates kainate receptors by interacting with the GluK2 subunit. Nat Commun. 2014;5:5182.

27. Modugno N, Lena F, Di Biasio F, Cerrone G, Ruggieri S, Fornai F. A clinical overview of non-motor symptoms in Parkinson's disease. Arch Ital Biol. 2013;151(4):148-68.

28. Bernal-Pacheco O, Limotai N, Go CL, Fernandez HH. Nonmotor manifestations in Parkinson disease. Neurologist. 2012;18(1):1-16.

29. Trempe JF, Sauve V, Grenier K, Seirafi M, Tang MY, Menade M, et al. Structure of parkin reveals mechanisms for ubiquitin ligase activation. Science. 2013;340(6139):1451-5.

30. Kumar A, Chaugule VK, Condos TEC, Barber KR, Johnson C, Toth R, et al Parkin-phosphoubiquitin complex reveals cryptic ubiquitin-binding site required for RBR ligase activity. Nat Struct Mol Biol. 2017;24(5):475-83.

31. Kumar A, Aguirre JD, Condos TE, Martinez-Torres RJ, Chaugule VK, Toth R, et al. Disruption of the autoinhibited state primes the E3 ligase parkin for activation and catalysis. EMBO J. 2015;34(20):2506-21.

32. Fiesel FC, Caulfield TR, Moussaud-Lamodiere EL, Ogaki K, Dourado DF, Flores SC, et al. Structural and functional impact of Parkinson disease-associated mutations in the E3 ubiquitin ligase Parkin. Hum Mutat. 2015;36(8):774-86.

33. Conceicao IC, Rama MM, Oliveira B, Cafe C, Almeida J, Mouga S, et al. Definition of a putative pathological region in PARK2 associated with autism spectrum disorder through in silico analysis of its functional structure. Psychiatr Genet. 2017;27(2):54-61.

34. Bekris LM, Mata IF, Zabetian CP. The genetics of Parkinson disease. J Geriatr Psychiatry Neurol. 2010;23(4):228-42.

35. Scherfler C, Khan NL, Pavese N, Eunson L, Graham E, Lees AJ, et al. Striatal and cortical pre- and postsynaptic dopaminergic dysfunction in sporadic parkin-linked parkinsonism. Brain. 2004;127(Pt 6):1332-42.

36. Foroud T, Uniacke SK, Liu L, Pankratz N, Rudolph A, Halter C, et al. Heterozygosity for a mutation in the Parkin gene leads to later onset Parkinson disease. Neurology. 2003;60(5):796-801.

37. Pankratz N, Kissell DK, Pauciulo MW, Halter CA, Rudolph A, Pfeiffer RF, et al Parkin dosage mutations have greater pathogenicity in familial PD than simple sequence mutations. Neurology. 2009;73(4):279-86.

38. Kreye J, Wenke NK, Chayka M, Leubner J, Murugan R, Maier N, et al. Human cerebrospinal fluid monoclonal $\mathrm{N}$-methyl-D-aspartate receptor autoantibodies are sufficient for encephalitis pathogenesis. Brain. 2016;139(Pt 10):2641-52.

39. Goo MS, Scudder SL, Patrick GN. Ubiquitin-dependent trafficking and turnover of ionotropic glutamate receptors. Front Mol Neurosci. 2015;8:60.

40. Widagdo J, Chai YJ, Ridder MC, Chau YQ, Johnson RC, Sah P, et al. Activitydependent ubiquitination of GluA1 and GluA2 regulates AMPA receptor intracellular sorting and degradation. Cell Rep. 2015;S2211-1247(15):00028-5.
41. Lussier MP, Sanz-Clemente A, Roche KW. Dynamic regulation of N-methyl-daspartate (NMDA) and alpha-Amino-3-hydroxy-5-methyl-4-

isoxazolepropionic acid (AMPA) receptors by posttranslational modifications. J Biol Chem. 2015:290(48):28596-603.

42. Bliss TV, Collingridge GL, Kaang BK, Zhuo M. Synaptic plasticity in the anterior cingulate cortex in acute and chronic pain. Nat Rev Neurosci. 2016; 17(8):485-96.

43. Pittenger $C$. Disorders of memory and plasticity in psychiatric disease. Dialogues Clin Neurosci. 2013;15(4):455-63.

44. Sweatt JD. Neural plasticity and behavior - sixty years of conceptual advances. J Neurochem. 2016;139(Suppl 2):179-99.

45. Luscher C, Malenka RC. NMDA receptor-dependent long-term potentiation and long-term depression (LTP/LTD). Cold Spring Harb Perspect Biol. 2012;4(6)

46. Nicoll RA. A brief history of long-term potentiation. Neuron. 2017;93(2):281-90.

47. Lee HK, Kameyama K, Huganir RL, Bear MF. NMDA induces long-term synaptic depression and dephosphorylation of the GluR1 subunit of AMPA receptors in hippocampus. Neuron. 1998;21(5):1151-62.

48. Lu W, Man H, Ju W, Trimble WS, MacDonald JF, Wang YT. Activation of synaptic NMDA receptors induces membrane insertion of new AMPA receptors and LTP in cultured hippocampal neurons. Neuron. 2001;29(1): 243-54

49. Pramstaller PP, Schlossmacher MG, Jacques TS, Scaravilli F, Eskelson C, Pepivani I, et al. Lewy body Parkinson's disease in a large pedigree with 77 Parkin mutation carriers. Ann Neurol. 2005;58(3):411-22.

50. Sun M, Latourelle JC, Wooten GF, Lew MF, Klein C, Shill HA, et al. Influence of heterozygosity for parkin mutation on onset age in familial Parkinson disease: the GenePD study. Arch Neurol. 2006;63(6):826-32

51. Kim CY, Alcalay RN. Genetic forms of Parkinson's disease. Semin Neurol. 2017:37(2):135-46.

52. Cremer JN, Amunts K, Schleicher A, Palomero-Gallagher N, Piel M, Rosch F, et al. Changes in the expression of neurotransmitter receptors in Parkin and DJ-1 knockout mice--a quantitative multireceptor study. Neuroscience. 2015 311:539-51.

53. Hanson JE, Orr AL, Madison DV. Altered hippocampal synaptic physiology in aged parkin-deficient mice. NeuroMolecular Med. 2010;12(3):270-6.

54. Khan NL, Graham E, Critchley P, Schrag AE, Wood NW, Lees AJ, et al. Parkin disease: a phenotypic study of a large case series. Brain. 2003;126(Pt 6): 1279-92.

55. Srivastava A, Tang MX, Mejia-Santana H, Rosado L, Louis ED, Caccappolo E, et al. The relation between depression and parkin genotype: the CORE-PD study. Parkinsonism Relat Disord. 2011;17(10):740-4.

56. Sharp ME, Caccappolo E, Mejia-Santana H, Tang MX, Rosado L, Orbe Reilly $M$, et al. The relationship between obsessive-compulsive symptoms and PARKIN genotype: the CORE-PD study. Mov Disord. 2015;30(2):278-83.

57. Murrough JW, Abdallah CG, Mathew SJ. Targeting glutamate signalling in depression: progress and prospects. Nat Rev Drug Discov. 2017;16(7):472-86.

58. Park M, Niciu MJ, Zarate CA Jr. Novel glutamatergic treatments for severe mood disorders. Curr Behav Neurosci Rep. 2015:2(4):198-208.

59. Feyissa AM, Chandran A, Stockmeier CA, Karolewicz B. Reduced levels of NR2A and NR2B subunits of NMDA receptor and PSD-95 in the prefrontal cortex in major depression. Prog Neuro-Psychopharmacol Biol Psychiatry. 2009;33(1):70-5

60. Kariuki-Nyuthe C, Gomez-Mancilla B, Stein DJ. Obsessive compulsive disorder and the glutamatergic system. Curr Opin Psychiatry. 2014;27(1):32-7.

61. Nishijima H, Ueno T, Funamizu Y, Ueno S, Tomiyama M. Levodopa treatment and dendritic spine pathology. Mov Disord. 2017;33(6):877-88.

62. Ueno T, Nishijima H, Ueno S, Tomiyama M. Spine enlargement of pyramidal tract-type neurons in the motor cortex of a rat model of levodopa-induced dyskinesia. Front Neurosci. 2017;11:206.

63. Prescott IA, Liu LD, Dostrovsky JO, Hodaie M, Lozano AM, Hutchison WD. Lack of depotentiation at basal ganglia output neurons in PD patients with levodopa-induced dyskinesia. Neurobiol Dis. 2014;71:24-33.

64. Scheuerle A, Wilson K. PARK2 copy number aberrations in two children presenting with autism spectrum disorder: further support of an association and possible evidence for a new microdeletion/microduplication syndrome. Am J Med Genet B Neuropsychiatr Genet. 2011;156B(4):413-20.

65. Roberts JL, Hovanes K, Dasouki M, Manzardo AM, Butler MG. Chromosomal microarray analysis of consecutive individuals with autism spectrum disorders or learning disability presenting for genetic services. Gene. 2014; 535(1):70-8 
66. Yin $\mathrm{CL}$, Chen HI, Li LH, Chien YL, Liao HM, Chou MC, et al. Genome-wide analysis of copy number variations identifies PARK2 as a candidate gene for autism spectrum disorder. Mol Autism. 2016;7:23.

67. Leal-Ortiz S, Waites CL, Terry-Lorenzo R, Zamorano P, Gundelfinger ED, Garner CC. Piccolo modulation of Synapsin1a dynamics regulates synaptic vesicle exocytosis. J Cell Biol. 2008;181(5):831-46.

68. Li D, Specht CG, Waites CL, Butler-Munro C, Leal-Ortiz S, Foote JW, et al. SAP97 directs NMDA receptor spine targeting and synaptic plasticity. J. Physiol. 2011;589(Pt 18):4491-510.

Ready to submit your research? Choose BMC and benefit from:

- fast, convenient online submission

- thorough peer review by experienced researchers in your field

- rapid publication on acceptance

- support for research data, including large and complex data types

- gold Open Access which fosters wider collaboration and increased citations

- maximum visibility for your research: over $100 \mathrm{M}$ website views per year

At $\mathrm{BMC}$, research is always in progress.

Learn more biomedcentral.com/submissions 